\title{
Nonlinear Elliptic Boundary Value Problems at Resonance with Nonlinear Wentzell Boundary Conditions
}

\author{
Ciprian G. Gal ${ }^{1}$ and Mahamadi Warma ${ }^{2}$ \\ ${ }^{1}$ Department of Mathematics, Florida International University, Miami, FL 33199, USA \\ ${ }^{2}$ Department of Mathematics, Faculty of Natural Sciences, University of Puerto Rico, Rio Piedras Campus, P.O. Box 70377, \\ San Juan, PR 00936-8377, USA \\ Correspondence should be addressed to Ciprian G. Gal; cgal@fiu.edu
}

Received 2 March 2017; Accepted 30 May 2017; Published 27 June 2017

Academic Editor: Luigi C. Berselli

Copyright (c) 2017 Ciprian G. Gal and Mahamadi Warma. This is an open access article distributed under the Creative Commons Attribution License, which permits unrestricted use, distribution, and reproduction in any medium, provided the original work is properly cited.

\begin{abstract}
Given a bounded domain $\Omega \subset \mathbb{R}^{N}$ with a Lipschitz boundary $\partial \Omega$ and $p, q \in(1,+\infty)$, we consider the quasilinear elliptic equation $-\Delta_{p} u+\alpha_{1}(u)=f$ in $\Omega$ complemented with the generalized Wentzell-Robin type boundary conditions of the form $b(x)|\nabla u|^{p-2} \partial_{\mathbf{n}} u-$ $\rho b(x) \Delta_{q, \Gamma} u+\alpha_{2}(u)=g$ on $\partial \Omega$. In the first part of the article, we give necessary and sufficient conditions in terms of the given functions $f, g$ and the nonlinearities $\alpha_{1}, \alpha_{2}$, for the solvability of the above nonlinear elliptic boundary value problems with the nonlinear boundary conditions. In other words, we establish a sort of "nonlinear Fredholm alternative" for our problem which extends the corresponding Landesman and Lazer result for elliptic problems with linear homogeneous boundary conditions. In the second part, we give some additional results on existence and uniqueness and we study the regularity of the weak solutions for these classes of nonlinear problems. More precisely, we show some global a priori estimates for these weak solutions in an $L^{\infty}$-setting.
\end{abstract}

Dedicated to the 70th birthday of Jerome A. Goldstein

\section{Introduction}

Let $\Omega \subset \mathbf{R}^{N}, N \geq 1$, be a bounded domain with a Lipschitz boundary $\partial \Omega$ and consider the following nonlinear boundary value problem with nonlinear second-order boundary conditions:

$$
\begin{aligned}
-\Delta_{p} u+\alpha_{1}(u)=f & \text { in } \Omega, \\
b(x)|\nabla u|^{p-2} \partial_{\mathbf{n}} u-\rho b(x) \Delta_{q, \Gamma} u+\alpha_{2}(u)=g & \text { on } \partial \Omega,
\end{aligned}
$$

where $b \in L^{\infty}(\partial \Omega), b(x) \geq b_{0}>0$, for some constant $b_{0}, \rho$ is either 0 or 1 , and $\alpha_{1}, \alpha_{2} \in C(\mathbb{R})$ are monotone nondecreasing functions such that $\alpha_{i}(0)=0$. Moreover, $\Delta_{p} u=\operatorname{div}\left(|\nabla u|^{p-2} \nabla u\right)$ is the $p$-Laplace operator, $p \in$ $(1,+\infty)$, and $f \in L^{2}(\Omega, d x), g \in L^{2}(\partial \Omega, \sigma)$ are given realvalued functions. Here, $d x$ denotes the usual $N$-dimensional Lebesgue measure in $\Omega$ and $\sigma$ denotes the restriction to $\partial \Omega$ of the $(N-1)$-dimensional Hausdorff measure. Recall that $\sigma$ coincides with the usual Lebesgue surface measure since $\Omega$ has a Lipschitz boundary, and $\partial_{n} u$ denotes the normal derivative of $u$ in direction of the outer normal vector $\overrightarrow{\mathbf{n}}$. Furthermore, $\Delta_{q, \Gamma}$ is defined as the generalized $q$-LaplaceBeltrami operator on $\partial \Omega$; that is, $\Delta_{q, \Gamma} u=\operatorname{div}_{\Gamma}\left(\left|\nabla_{\Gamma} u\right|^{q-2} \nabla_{\Gamma} u\right)$, $q \in(1,+\infty)$. In particular, $\Delta_{2}=\Delta$ and $\Delta_{2, \Gamma}=\Delta_{\Gamma}$ become the well-known Laplace and Laplace-Beltrami operators on $\Omega$ and $\partial \Omega$, respectively. Here, for any real-valued function $v$,

$$
\operatorname{div}_{\Gamma} v=\sum_{i=1}^{N-1} \partial_{\tau_{i}} v,
$$

where $\partial_{\tau_{i}} v$ denotes the directional derivative of $v$ along the tangential directions $\tau_{i}$ at each point on the boundary, whereas $\nabla_{\Gamma} v=\left(\partial_{\tau_{1}} v, \ldots, \partial_{\tau_{N-1}} v\right)$ denotes the tangential gradient at $\partial \Omega$. It is worth mentioning again that when 
$\rho=0$ in (1), the boundary conditions are of lower order than the order of the $p$-Laplace operator, while, for $\rho=$ 1 , we deal with boundary conditions which have the same differential order as the operator acting in the domain $\Omega$. Such boundary conditions arise in many applications, such as phase-transition phenomena (see, e.g., [1, 2] and the references therein), and have been studied by several authors (see, e.g., [3-7]).

In [4], the authors have formulated necessary and sufficient conditions for the solvability of (1) when $p=q=2$, by establishing a sort of "nonlinear Fredholm alternative" for such elliptic boundary value problems. We shall now state their main result. Defining two real parameters $\lambda_{1}, \lambda_{2} \in \mathbb{R}_{+}$ by

$$
\begin{aligned}
& \lambda_{1}=\int_{\Omega} d x, \\
& \lambda_{2}=\int_{\partial \Omega} \frac{d \sigma}{b},
\end{aligned}
$$

this result reads that a necessary condition for the existence of a weak solution of (1) is that

$$
\begin{array}{r}
\int_{\Omega} f(x) d x+\int_{\partial \Omega} g(x) \frac{d \sigma}{b(x)} \\
\in\left(\lambda_{1} \mathscr{R}\left(\alpha_{1}\right)+\lambda_{2} \mathscr{R}\left(\alpha_{2}\right)\right),
\end{array}
$$

while a sufficient condition is

$$
\begin{aligned}
& \int_{\Omega} f(x) d x+\int_{\partial \Omega} g(x) \frac{d \sigma}{b(x)} \\
& \quad \in \operatorname{int}\left(\lambda_{1} \mathscr{R}\left(\alpha_{1}\right)+\lambda_{2} \mathscr{R}\left(\alpha_{2}\right)\right),
\end{aligned}
$$

where $\mathscr{R}\left(\alpha_{j}\right)$ denotes the range of $\alpha_{j}, j=1,2$, and $\operatorname{int}(G)$ denotes the interior of the set $G$.

Relation (4) turns out to be both necessary and sufficient if either of the sets $\mathscr{R}\left(\alpha_{1}\right)$ or $\mathscr{R}\left(\alpha_{2}\right)$ is an open interval. This particular result was established in [4, Theorem 3], by employing methods from convex analysis involving subdifferentials of convex, lower semicontinuous functionals on suitable Hilbert spaces. As an application of our results, we can consider the following boundary value problem:

$$
\begin{aligned}
-\Delta u+\alpha_{1}(u)=f & \text { in } \Omega, \\
b(x) \partial_{n} u=g & \text { on } \partial \Omega,
\end{aligned}
$$

which is only a special case of (1) (i.e., $\rho=0, \alpha_{2} \equiv 0$, and $p=$ 2 ). According to [4, Theorem 3] (see also (5)), this problem has a weak solution if

$$
\int_{\Omega} f(x) d x+\int_{\partial \Omega} g(x) \frac{d \sigma}{b(x)} \in \operatorname{int}\left(\lambda_{1} \mathscr{R}\left(\alpha_{1}\right)\right),
$$

which yields the result of Landesman and Lazer [8] for $g \equiv 0$. This last condition is both necessary and sufficient when the interval $\mathscr{R}\left(\alpha_{1}\right)$ is open. This was put into an abstract context and significantly extended by Brézis and Haraux [9]. Their work was much further extended by Brézis and Nirenberg [10]. The goal of the present article is comparable to that of [4] since we want to establish similar conditions to (5) and (7) for the existence of solutions to (1) when $p, q \neq 2$, with main emphasis on the generality of the boundary conditions.

Recall that $\lambda_{1}$ and $\lambda_{2}$ are given by (3). Let $\square$ be the interval $\lambda_{1} \mathscr{R}\left(\alpha_{1}\right)+\lambda_{2} \mathscr{R}\left(\alpha_{2}\right)$. Our first main result is as follows (see Section 4 also).

Theorem 1. Let $\alpha_{j}: \mathbb{R} \rightarrow \mathbb{R}(j=1,2)$ be odd, monotone nondecreasing, continuous function such that $\alpha_{j}(0)=0$. Assume that the functions $\Lambda_{j}(t):=\int_{0}^{|t|} \alpha_{j}(s) d s$ satisfy

$$
\Lambda_{j}(2 t) \leq C_{j} \Lambda_{j}(t), \quad \forall t \in \mathbb{R},
$$

for some constants $C_{j}>1, j=1,2$. If $u$ is a weak solution of (1) (in the sense of Definition 30 below), then

$$
\int_{\Omega} f(x) d x+\int_{\partial \Omega} g(x) \frac{d \sigma}{b(x)} \in \mathbb{\square} .
$$

Conversely, if

$$
\int_{\Omega} f(x) d x+\int_{\partial \Omega} g(x) \frac{d \sigma}{b(x)} \in \operatorname{int}(\llbracket),
$$

then (1) has a weak solution.

Our second main result of the paper deals with a modified version of (1) which is obtained by replacing the functions $\alpha_{1}(s)$ and $\alpha_{2}(s)$ in $(1)$ by $\bar{\alpha}_{1}(s)+|s|^{p-2} s$ and $\bar{\alpha}_{2}(s)+\rho b|u|^{q-2} u$, respectively, and also allowing $\bar{\alpha}_{1}, \bar{\alpha}_{2}$ to depend on $x \in \bar{\Omega}$. Under additional assumptions on $\bar{\alpha}_{1}, \bar{\alpha}_{2}$ and under higher integrability properties for the data $(f, g)$, the next theorem provides us with conditions for unique solvability results for solutions to such boundary value problems. Then, we obtain some regularity results for these solutions. In addition to these results, the continuous dependence of the solution to (1) with respect to the data $(f, g)$ can be also established. In particular, we prove the following.

Theorem 2. Let all the assumptions of Theorem 1 be satisfied for the functions $\bar{\alpha}_{1}, \bar{\alpha}_{2}$. Moreover, for each $j=1,2$, assume that $\bar{\alpha}_{j}(t) / t \rightarrow 0$, as $t \rightarrow 0$, and $\bar{\alpha}_{j}(t) / t \rightarrow \infty$, as $t \rightarrow \infty$, respectively.

(a) Then, for every $(f, g) \in L^{p_{1}}(\Omega) \times L^{q_{1}}(\partial \Omega)$ with

$$
\begin{array}{ll}
p_{1}>\max \left\{1, \frac{N}{p}\right\}, & \begin{cases}\max \left\{1, \frac{N-1}{p-1}\right\}, & \text { if } \rho \in\{0,1\}, \\
q_{1}>\left\{\max \left\{1, \frac{N-1}{p}\right\},\right. & \text { if } \rho=1, p=q,\end{cases}
\end{array}
$$

there exists a unique weak solution to problem (1) (in the sense of Definition 40 below) which is bounded.

(b) Let $\bar{\alpha}_{j}, j=1,2$, be such that

$$
c_{j}\left|\bar{\alpha}_{j}(\xi-\eta)\right| \leq\left|\bar{\alpha}_{j}(\xi)-\bar{\alpha}_{j}(\eta)\right|, \quad \forall \xi, \eta \in \mathbb{R},
$$


for some constants $c_{j} \in(0,1]$. Then, the weak (bounded) solution of problem (1) depends continuously on the data $(f, g)$. Precisely, let us indicate by $u_{F_{j}}$ the unique solution corresponding to the data $F_{j}:=$ $\left(f_{j}, g_{j}\right) \in L^{p_{1}}(\Omega) \times L^{q_{1}}(\partial \Omega)$, for each $j=1,2$. Then, the following estimate holds:

$$
\begin{aligned}
& \left\|u_{F_{1}}-u_{F_{2}}\right\|_{L^{\infty}(\Omega)}+\left\|u_{F_{1}}-u_{F_{2}}\right\|_{L^{\infty}(\partial \Omega)} \\
& \quad \leq Q\left(\left\|f_{1}-f_{2}\right\|_{L^{p_{1}}(\Omega)},\left\|g_{1}-g_{2}\right\|_{L^{q_{1}}(\partial \Omega)}\right),
\end{aligned}
$$

for some nonnegative function $Q: \mathbb{R}_{+}^{2} \rightarrow \mathbb{R}_{+}$, $Q(0,0)=0$, which can be computed explicitly.

We organize the paper as follows. In Section 2, we introduce some notations and recall some well-known results about Sobolev spaces, maximal monotone operators, and Orlicz type spaces which will be needed throughout the article. In Section 3, we show that the subdifferential of a suitable functional associated with problem (1) satisfies a sort of "quasilinear" version of the Fredholm alternative (cf. Theorem 20), which is needed in order to obtain the result in Theorem 1. Finally, in Sections 4 and 5, we provide detailed proofs of Theorems 1 and 2. We also illustrate the application of these results with some examples.

\section{Preliminaries and Notations}

In this section we put together some well-known results on nonlinear forms, maximal monotone operators, and Sobolev spaces. For more details on maximal monotone operators, we refer to the monographs [11-15]. We will also introduce some notations.

2.1. Maximal Monotone Operators. Let $H$ be a real Hilbert space with scalar product $(\cdot, \cdot)_{H}$.

Definition 3. Let $A: D(A) \subset H \rightarrow H$ be a closed (nonlinear) operator. The operator $A$ is said to be

(i) monotone if for all $u, v \in D(A)$ one has

$$
(A u-A v, u-v)_{H} \geq 0,
$$

(ii) maximal monotone if it is monotone and the operator $I+A$ is invertible.

Next, let $V$ be a real reflexive Banach space which is densely and continuously embedded into the real Hilbert space $H$, and let $V^{\prime}$ be its dual space such that $V \hookrightarrow H \hookrightarrow V^{\prime}$.

Definition 4. Let $\mathscr{A}: V \times V \rightarrow \mathbb{R}$ be a continuous map.

(a) The map $\mathscr{A}: V \times V \rightarrow \mathbb{R}$ is called a nonlinear form on $H$ if for all $u \in V$ one has $\mathscr{A}(u, \cdot) \in V^{\prime}$, that is, if $\mathscr{A}$ is linear and bounded in the second variable.

(b) The nonlinear form $\mathscr{A}: V \times V \rightarrow \mathbb{R}$ is said to be

(i) monotone if $\mathscr{A}(u, u-v)-\mathscr{A}(v, u-v) \geq$ 0 for all $u, v \in V$; (ii) hemicontinuous if $\lim _{t \downarrow 0} \mathscr{A}(u+t v, w)=\mathscr{A}(u, w)$, $\forall u, v, w \in V$

(iii) coercive, if $\lim _{\|v\|_{V} \rightarrow+\infty}\left(\mathscr{A}(v, v) /\|v\|_{V}\right)=+\infty$.

Now, let $\varphi: H \rightarrow(-\infty,+\infty$ ] be a proper, convex, lower semicontinuous functional with effective domain

$$
D(\varphi):=\{u \in H: \varphi(u)<\infty\} .
$$

The subdifferential $\partial \varphi$ of the functional $\varphi$ is defined by

$$
\begin{aligned}
& D(\partial \varphi):=\{u \in D(\varphi): \exists w \in H, \forall v \in D(\varphi): \varphi(v) \\
& \left.\quad-\varphi(u) \geq(w, v-u)_{H}\right\} ; \\
& \partial \varphi(u):=\{w \in H: \forall v \in D(\varphi): \varphi(v)-\varphi(u) \\
& \left.\quad \geq(w, v-u)_{H}\right\} .
\end{aligned}
$$

By a classical result of Minty [13] (see also [12, 14]), $\partial \varphi$ is a maximal monotone operator.

2.2. Functional Setup. Let $\Omega \subset \mathbb{R}^{N}$ be a bounded domain with a Lipschitz boundary $\partial \Omega$. For $1<p<\infty$, we let $W^{1, p}(\Omega)$ be the first-order Sobolev space; that is,

$$
W^{1, p}(\Omega)=\left\{u \in L^{p}(\Omega): \nabla u \in\left(L^{p}(\Omega)\right)^{N}\right\} .
$$

Then $W^{1, p}(\Omega)$, endowed with the norm

$$
\|u\|_{W^{1, p}(\Omega)}:=\left(\|u\|_{\Omega, p}^{p}+\|\nabla u\|_{\Omega, p}^{p}\right)^{1 / p},
$$

is a Banach space, where we have set

$$
\|u\|_{\Omega, p}^{p}:=\int_{\Omega}|u|^{p} d x .
$$

Since $\Omega$ has a Lipschitz boundary, it is well-known that there exists a constant $C>0$ such that

$$
\|u\|_{\Omega, p_{s}} \leq C\|u\|_{W^{1, p}(\Omega)}, \quad \forall u \in W^{1, p}(\Omega)
$$

where $p_{s}=p N /(N-p)$ if $p<N$ and $1 \leq p_{s}<\infty$ if $N=p$. Moreover the trace operator $\operatorname{tr}(u):=u_{\left.\right|_{\partial \Omega}}$ initially defined for $u \in C^{1}(\bar{\Omega})$ has an extension to a bounded linear operator from $W^{1, p}(\Omega)$ into $L^{q_{s}}(\partial \Omega)$ where $q_{s}:=p(N-1) /(N-p)$ if $p<N$ and $1 \leq q_{s}<\infty$ if $N=p$. Hence, there is a constant $C>0$ such that

$$
\|u\|_{\partial \Omega, q_{s}} \leq C\|u\|_{W^{1, p}(\Omega)}, \quad \forall u \in W^{1, p}(\Omega) .
$$

Throughout the remainder of this article, for $1<p<N$, we let

$$
\begin{aligned}
& p_{s}:=\frac{p N}{N-p}, \\
& q_{s}:=\frac{p(N-1)}{N-p} .
\end{aligned}
$$


If $p>N$, one has that

$$
W^{1, p}(\Omega) \hookrightarrow C^{0,1-N / p}(\bar{\Omega}) ;
$$

that is, the space $W^{1, p}(\Omega)$ is continuously embedded into $C^{0,1-N / p}(\bar{\Omega})$. For more details, we refer to [16, Theorem 4.7] (see also [17, Chapter 4]).

For $1<q<\infty$, we define the Sobolev space $W^{1, q}(\partial \Omega)$ to be the completion of the space $C^{1}(\partial \Omega)$ with respect to the norm

$$
\|u\|_{W^{1, q}(\partial \Omega)}:=\left(\int_{\partial \Omega}|u|^{q} d \sigma+\int_{\partial \Omega}\left|\nabla_{\Gamma} u\right|^{q} d \sigma\right)^{1 / q},
$$

where we recall that $\nabla_{\Gamma} u$ denotes the tangential gradient of the function $u$ at the boundary $\partial \Omega$. It is also well-known that $W^{1, q}(\partial \Omega)$ is continuously embedded into $L^{q_{t}}(\partial \Omega)$ where $q_{t}:=$ $q(N-1) /(N-1-q)$ if $1<q<N-1$ and $1 \leq q_{t}<\infty$ if $q=N-1$. Hence, for $1<q \leq N-1$, there exists a constant $C>0$ such that

$$
\|u\|_{q_{t}, \partial \Omega} \leq C\|u\|_{W^{1, q}(\partial \Omega)}, \quad \forall u \in W^{1, q}(\partial \Omega) .
$$

Let $\lambda_{N}$ denote the $N$-dimensional Lebesgue measure and let the measure $\mu:=\left.\lambda_{N}\right|_{\Omega} \oplus \sigma$ on $\bar{\Omega}$ be defined for every measurable set $A \subset \bar{\Omega}$ by

$$
\mu(A):=\lambda_{N}(\Omega \cap A)+\sigma(A \cap \partial \Omega) .
$$

For $p, q \in[1, \infty]$, we define the Banach space

$$
\begin{aligned}
X^{p, q} & (\bar{\Omega}, \mu) \\
& :=\left\{F=(f, g): f \in L^{p}(\Omega), g \in L^{q}(\partial \Omega)\right\},
\end{aligned}
$$

endowed with the norm

$$
\|F\|_{X^{p, q}(\bar{\Omega})}=\|\mid F\|_{p, q}:=\|f\|_{\Omega, p}+\|g\|_{\partial \Omega, q},
$$

if $1 \leq p, q<\infty$, and

$$
\|F\|_{X^{\infty, \infty}(\bar{\Omega}, \mu)}=\|\mid F\|_{\infty}:=\max \left\{\|f\|_{\Omega, \infty},\|g\|_{\partial \Omega, \infty}\right\} .
$$

If $p=q$, we will simply denote $\left\|\left|F\|\|_{p, p}=\|\mid F\|_{p}\right.\right.$.

Identifying each function $u \in W^{1, p}(\Omega)$ with $U=$ $\left(u,\left.u\right|_{\partial \Omega}\right)$, we have that $W^{1, p}(\Omega)$ is a subspace of $X^{p, p}(\bar{\Omega}, \mu)$.

For $1<p, q<\infty$, we endow

$$
\begin{aligned}
\mathscr{V}_{1} & :=\left\{U:=\left(u,\left.u\right|_{\partial \Omega}\right), u \in W^{1, p}(\Omega),\left.u\right|_{\partial \Omega}\right. \\
& \left.\in W^{1, q}(\partial \Omega)\right\},
\end{aligned}
$$

with the norm

$$
\|U\|_{\mathscr{V}_{1}}:=\|u\|_{W^{1, p}(\Omega)}+\|u\|_{W^{1, q}(\partial \Omega)},
$$

while

$$
\mathscr{V}_{0}:=\left\{U=\left(u,\left.u\right|_{\partial \Omega}\right): u \in W^{1, p}(\Omega)\right\}
$$

is endowed with the norm

$$
\|U\|_{\mathscr{V}_{0}}:=\|u\|_{W^{1, p}(\Omega)} .
$$

It follows from (21) and (22) that $\mathscr{V}_{0}$ is continuously embedded into $X^{p_{s}, q_{s}}(\bar{\Omega}, \mu)$, with $p_{s}$ and $q_{s}$ given by (23), for $1<p<$ $N$. Moreover, by (21) and (26), $\mathscr{V}_{1}$ is continuously embedded into $X^{p_{s}, q_{t}}(\bar{\Omega}, \mu)$.
2.3. Musielak-Orlicz Type Spaces. For the convenience of the reader, we introduce the Orlicz and Musielak-Orlicz type spaces and prove some properties of these spaces which will be frequently used in the sequel (see Section 5).

Definition 5. Let $(X, \Sigma, v)$ be a complete measure space. We call a function $B: X \times \mathbb{R} \rightarrow[0, \infty]$ a Musielak-Orlicz function on $X$ if

(a) $B(x, \cdot)$ is nontrivial, even, and convex for $v$, a.e. $x \in X$;

(b) $B(x, \cdot)$ is vanishing and continuous at 0 for $v$, a.e. $x \in$ $X$;

(c) $B(x, \cdot)$ is left continuous on $[0, \infty)$;

(d) $B(\cdot, t)$ is $\sum$-measurable for all $t \in[0, \infty)$;

(e) $\lim _{t \rightarrow \infty}(B(x, t) / t)=\infty$. by

The complementary Musielak-Orlicz function $\widetilde{B}$ is defined

$$
\widetilde{B}(x, t):=\sup \{s|t|-B(x, s): s>0\} .
$$

It follows directly from the definition that for $t, s \geq 0$ (and hence for all $t, s \in \mathbb{R}$ )

$$
s t \leq B(x, t)+\widetilde{B}(x, s) .
$$

Definition 6. We say that a Musielak-Orlicz function $B$ satisfies the $\left(\triangle_{\alpha}^{0}\right)$-condition $(\alpha>1)$ if there exists a set $X_{0}$ of $\nu$-measure zero and a constant $C_{\alpha}>1$ such that

$$
B(x, \alpha t) \leq C_{\alpha} B(x, t),
$$

for all $t \in \mathbb{R}$ and every $x \in X \backslash X_{0}$.

We say that $B$ satisfies the $\left(\nabla_{2}^{0}\right)$-condition if there is a set $X_{0}$ of $\nu$-measure zero and a constant $c>1$ such that

$$
B(x, t) \leq \frac{1}{2 c} B(x, c t),
$$

for all $t \in \mathbb{R}$ and all $x \in X \backslash X_{0}$.

Definition 7. A function $\Phi: \mathbb{R} \rightarrow[0, \infty)$ is called an $\mathcal{N}$ function if

(i) $\Phi$ is even, strictly increasing, and convex;

(ii) $\Phi(t)=0$ if and only if $t=0$;

(iii) $\lim _{t \rightarrow 0}(\Phi(t) / t)=0$ and $\lim _{t \rightarrow \infty}(\Phi(t) / t)=\infty$.

We say that an $\mathcal{N}$-function $\Phi$ satisfies the $\left(\triangle_{2}\right)$-condition if there exists a constant $C_{2}>1$ such that

$$
\Phi(2 t) \leq C_{2} \Phi(t), \quad \forall t \in \mathbb{R}
$$

and it satisfies the $\left(\nabla_{2}\right)$-condition if there is a constant $c>1$ such that

$$
\Phi(t) \leq \frac{\Phi(c t)}{(2 c)}, \quad \forall t \in \mathbb{R} .
$$

For more details on $\mathscr{N}$-functions, we refer to the monograph of Adams [18, Chapter VIII] (see also [19, Chapter I], [20, Chapter I]). 
Remark 8. For an $\mathcal{N}$-function $\Phi$, we let $\varphi$ be its leftsided derivative. Then $\varphi$ is left continuous on $(0, \infty)$ and nondecreasing. Let $\psi$ be given by

$$
\psi(s):=\inf \{t>0: \varphi(t)>s\} .
$$

Then

$$
\begin{aligned}
& \Phi(t)=\int_{0}^{|t|} \varphi(s) d s \\
& \Psi(t):=\int_{0}^{|t|} \psi(s) d s=\sup \{|t| s-\Phi(s): s>0\} .
\end{aligned}
$$

As before for all $s, t \in \mathbb{R}$

$$
s t \leq \Phi(t)+\Psi(s) .
$$

Moreover, if $s=\varphi(t)$ or $t=\psi(s)$ then we have equality; that is,

$$
\Psi(\varphi(t))=t \varphi(t)-\Phi(t)
$$

The function $\Psi$ is called the complementary $\mathcal{N}$-function of $\Phi$. It is also known that an $\mathcal{N}$-function $\Phi$ satisfies the $\left(\triangle_{2}\right)$ condition if and only if

$$
\operatorname{ct} \varphi(t) \leq \Phi(t) \leq t \varphi(t),
$$

for some constant $c \in(0,1]$ and for all $t \in \mathbb{R}$, where $\varphi$ is the left-sided derivative of $\Phi$.

Lemma 9. Let $\Phi$ be an $\mathcal{N}$-function which satisfies the $\left(\triangle_{2}\right)$ condition with the constant $C_{2}>1$ and let $\Psi$ be its complementary $\mathcal{N}$-function. Then $\Psi$ satisfies the $\left(\nabla_{2}\right)$-condition with the constant $c:=2^{C_{2}-1}$.

Proof. We have

$$
\begin{aligned}
t \varphi(t) & \leq \int_{t}^{2 t} \varphi(s) d s \leq \int_{0}^{2 t} \varphi(s) d s=\Phi(2 t) \\
& \leq C_{2} \Phi(t) .
\end{aligned}
$$

Since $\varphi(\psi(s)) \geq s$ for all $s \geq 0$ and $s / \Psi(s)$ and $s /(s-1)$ are decreasing, we get, for $t:=\psi(s)$, that

$$
\begin{aligned}
\frac{s \psi(s)}{\Psi(s)} & \geq \frac{\varphi(\psi(s)) \psi(s)}{\Psi(\varphi(\psi(s)))}=\frac{t \varphi(t)}{\Psi(\varphi(t))}=\frac{t \varphi(t)}{t \varphi(t)-\Phi(t)} \\
& \geq \frac{C_{2}}{C_{2}-1} .
\end{aligned}
$$

Now let $c:=2^{C_{2}-1}$. Then for $t \geq 0$

$$
\begin{aligned}
\ln \left(\frac{\Psi(c t)}{\Psi(t)}\right) & =\int_{t}^{c t} \frac{\Psi(s)}{\Psi(s)} d s \geq \int_{t}^{c t} \frac{C_{2}}{s\left(C_{2}-1\right)} d s \\
& =\frac{C_{2}}{C_{2}-1} \ln (c)=C_{2} \log (2) \\
& =\ln \left(2 \cdot 2^{C_{2}-1}\right) .
\end{aligned}
$$

Hence, $\Psi(t) 2 c \leq \Psi(c t)$.
Corollary 10. Let $B$ be a Musielak-Orlicz function such that $B(x, \cdot)$ is an $\mathcal{N}$-function for $v$, a.e. on $x$. If $B$ satisfies the $\left(\triangle_{2}^{0}\right)$ condition, then $\widetilde{B}$ satisfies the $\left(\nabla_{2}^{0}\right)$-condition.

Definition 11. Let $B$ be a Musielak-Orlicz function. Then the Musielak-Orlicz space $L^{B}(X)$ associated with $B$ is defined by

$$
\begin{aligned}
& L^{B}(X):=\left\{u: X \longrightarrow \mathbb{R} \text { measurable: } \rho_{B}\left(\frac{u}{\alpha}\right)\right. \\
& \quad<\infty \text { for some } \alpha>0\},
\end{aligned}
$$

where

$$
\rho_{B}(v):=\int_{X} B(x, v(x)) d v(x)
$$

On this space we consider the Luxemburg norm $\|\cdot\|_{X, B}$ defined by

$$
\|u\|_{X, B}:=\inf \left\{\alpha>0: \rho_{B}\left(\frac{u}{\alpha}\right) \leq 1\right\} .
$$

Proposition 12. Let $B$ be a Musielak-Orlicz function which satisfies the $\left(\nabla_{2}^{0}\right)$-condition. Then

$$
\lim _{\|u\|_{X, B} \rightarrow+\infty} \frac{\rho_{B}(u)}{\|u\|_{X, B}}=+\infty .
$$

Proof. If $B$ satisfies the $\left(\nabla_{2}^{0}\right)$-condition, then there exists a set $X_{0} \subset X$ of measure zero such that for every $\varepsilon>0$ there exists $\alpha=\alpha(\varepsilon)>0$

$$
B(x, \alpha t) \leq \alpha \varepsilon B(x, t)
$$

for all $t \in \mathbb{R}$ and all $x \in X \backslash X_{0}$. Let $\lambda \in(0, \infty)$ be fixed. For $\varepsilon:=1 / \lambda$ there exists $\alpha>0$ satisfying the above inequality. We will show that $\rho_{B}(u) \geq \lambda\|u\|_{X, B}$ whenever $\|u\|_{X, B}>1 / \alpha$. Assume that $\|u\|_{X, B}>1 / \alpha$ and let $\delta>0$ be such that $\alpha=$ $(1+\delta) /\|u\|_{X, B}$. Then

$$
\begin{aligned}
\rho_{B}(\alpha u) & =\int_{X} B\left(x, \frac{u(1+\delta)}{\|u\|_{X, B}}\right) d \mu \\
& \geq(1+\delta)^{1-1 / n} \int_{X} B\left(x, \frac{u(1+\delta)^{1 / n}}{\|u\|_{X, B}}\right) d \mu \\
& \geq(1+\delta)^{1-1 / n},
\end{aligned}
$$

for all $n \in \mathbb{N}$. If we assume that the last inequality does not hold, then

$$
\frac{\|u\|_{X, B}}{(1+\delta)} \in\left\{\alpha>0: \rho\left(\frac{u}{\alpha}\right) \leq 1\right\}
$$


and this clearly contradicts the definition of $\|u\|_{X, B}$. Therefore, we must have

$$
\rho_{B}(\alpha u) \geq 1+\delta=\alpha\|u\|_{X, B}
$$

From (53), (56), we obtain

$$
\begin{aligned}
\rho_{B}(u) & =\int_{X} B(x, u(x)) d \mu \geq \frac{\lambda}{\alpha} \int_{X} B(x, \alpha u(x)) d \mu \\
& =\frac{\lambda}{\alpha} \rho_{B}(\alpha u) \geq \lambda\|u\|_{X, B} .
\end{aligned}
$$

The proof is finished.

Corollary 13. Let B be a Musielak-Orlicz function such that $B(x, \cdot)$ is an $\mathcal{N}$-function for $v$, a.e. on $x$. If its complementary $\mathcal{N}$-function $\widetilde{B}$ satisfies the $\left(\triangle_{2}^{0}\right)$-condition, then $B$ satisfies the $\left(\nabla_{2}^{0}\right)$-condition and

$$
\lim _{\|u\|_{X, B} \rightarrow+\infty} \frac{\rho_{B}(u)}{\|u\|_{X, B}}=+\infty
$$

2.4. Some Tools. For the reader's convenience, we report here below some useful inequalities which will be needed in the course of investigation.

Lemma 14. Let $a, b \in \mathbb{R}^{N}$ and $p \in(1, \infty)$. Then, there exists a constant $C_{p}>0$ such that

$$
\begin{aligned}
& \left(|a|^{p-2} a-|b|^{p-2} b\right)(a-b) \\
& \quad \geq C_{p}(|a|+|b|)^{p-2}|a-b|^{2} \geq 0 .
\end{aligned}
$$

If $p \in[2, \infty)$, then there exists a constant $c_{p} \in(0,1]$ such that

$$
\left(|a|^{p-2} a-|b|^{p-2} b\right)(a-b) \geq c_{p}|a-b|^{p} .
$$

Proof. The proof of (60) is included in [21, Lemma I.4.4]. In order to show (59), one only needs to show that the left hand side is nonnegative, which follows easily.

The following result which is of analytic nature and whose proof can be found in [22, Lemma 3.11] will be useful in deriving some a priori estimates of weak solutions of elliptic equations.

Lemma 15. Let $\psi:\left[k_{0}, \infty\right) \rightarrow \mathbb{R}$ be a nonnegative, nonincreasing function such that there are positive constants $c, \alpha$ and $\delta(\delta>1)$ such that

$$
\psi(h) \leq c(h-k)^{-\alpha} \psi(k)^{\delta}, \quad \forall h>k \geq k_{0} .
$$

Then $\psi\left(k_{0}+d\right)=0$ with $d=c^{1 / \alpha} \psi\left(k_{0}\right)^{(\delta-1) / \alpha} 2^{\delta(\delta-1)}$.

\section{The Fredholm Alternative}

In what follows, we assume that $\Omega \subset \mathbb{R}^{N}$ is a bounded domain with Lipschitz boundary $\partial \Omega$. Let $b \in L^{\infty}(\partial \Omega)$ satisfy $b(x) \geq b_{0}>0$ for some constant $b_{0}$. Let $\mathbb{X}_{2}$ be the real Hilbert space $L^{2}(\Omega, d x) \oplus L^{2}(\partial \Omega, d \sigma / b)$. Then, it is clear that $\mathbb{X}_{2}$ is isomorphic to $X^{2,2}\left(\bar{\Omega}, \lambda_{N} \oplus \sigma\right)$ with equivalent norms.

Next, let $\rho \in\{0,1\}$ and $p, q \in(1,+\infty)$ be fixed. We define the functional $\mathscr{J}_{\rho}: \mathbb{X}_{2} \rightarrow[0,+\infty]$ by setting

$$
\mathscr{F}_{\rho}(U)= \begin{cases}\frac{1}{p} \int_{\Omega}|\nabla u|^{p} d x+\frac{1}{q} \int_{\partial \Omega} \rho\left|\nabla_{\Gamma} u\right|^{q} d \sigma, & \text { if } U=\left(u, u_{\mid \partial \Omega}\right) \in D\left(\mathscr{F}_{\rho}\right), \\ +\infty, & \text { if } U \in \mathbb{X}_{2} \backslash D\left(\mathscr{F}_{\rho}\right),\end{cases}
$$

where the effective domain is given by $D\left(\mathscr{J}_{\rho}\right)=\mathscr{V}_{\rho} \cap \mathbb{X}_{2}$.

Throughout the remainder of this section, we let $\mu:=\lambda_{N} \oplus$ $d \sigma / b$. The following result can be obtained easily.

Proposition 16. The functional $\mathscr{J}_{\rho}$ defined by (62) is proper, convex, and lower semicontinuous on $\mathbb{X}_{2}=X^{2,2}(\bar{\Omega}, \mu)$.

The following result contains a computation of the subdifferential $\partial \mathscr{J}_{\rho}$ for the functional $\mathscr{J}_{\rho}$.

Remark 17. Let $U=\left(u,\left.u\right|_{\partial \Omega}\right) \in D\left(\mathscr{F}_{\rho}\right)$ and let $F:=(f, g) \in$ $\partial \mathscr{J}_{\rho}(U)$. Then, by definition, $F \in \mathbb{X}_{2}$ and, for all $V=$ $\left(v,\left.v\right|_{\partial \Omega}\right) \in D\left(\mathscr{J}_{\rho}\right)$, we have

$$
\begin{aligned}
\int_{\bar{\Omega}} F(V-U) d \mu \leq & \frac{1}{p} \int_{\Omega}\left(|\nabla v|^{p}-|\nabla u|^{p}\right) d x \\
& +\frac{1}{q} \rho \int_{\Omega}\left(\left|\nabla_{\Gamma} v\right|^{q}-\left|\nabla_{\Gamma} u\right|^{q}\right) d \sigma
\end{aligned}
$$

Let $W=\left(w,\left.w\right|_{\partial \Omega}\right) \in D\left(\mathscr{J}_{\rho}\right), 0<t \leq 1$, and set $V:=t W+U$ above. Dividing by $t$ and taking the limit as $t \downarrow 0$, we obtain that

$$
\begin{aligned}
\int_{\bar{\Omega}} F W d \mu \leq & \int_{\Omega}|\nabla u|^{p-2} \nabla u \cdot \nabla w d x \\
& +\rho \int_{\partial \Omega}\left|\nabla_{\Gamma}\right|^{q-2} \nabla_{\Gamma} u \cdot \nabla_{\Gamma} w d \sigma,
\end{aligned}
$$

where we recall that

$$
\int_{\bar{\Omega}} F d \mu=\int_{\Omega} f d x+\int_{\partial \Omega} g \frac{d \sigma}{b} .
$$

Choosing $w= \pm \psi$ with $\psi \in \mathscr{D}(\Omega)$ (the space of test functions) and integrating by parts in (64), we obtain

$$
\begin{aligned}
-\Delta_{p} u & =f \quad \text { in } \mathscr{D}^{\prime}(\Omega), \\
g & =b(x)|\nabla u|^{p-2} \partial_{n} u-\rho b(x) \Delta_{q, \Gamma} u
\end{aligned}
$$


Therefore, the single-valued operator $\partial \mathscr{F}_{\rho}$ is given by

$$
\begin{aligned}
& D\left(\partial_{\rho}\right)=\left\{U=\left(u, u_{\mid \partial \Omega}\right) \in D\left(\mathscr{J}_{\rho}\right),\right. \\
& \left.\left(-\Delta_{p} u, b(x)|\nabla u|^{p-2} \partial_{n} u-\rho b(x) \Delta_{q, \Gamma} u\right) \in \mathbb{X}_{2}\right\}, \\
& \partial \mathscr{J}_{\rho}(U)=\left(-\Delta_{p} u, b(x)|\nabla u|^{p-2} \partial_{n} u-\rho b(x) \Delta_{q, \Gamma} u\right) .
\end{aligned}
$$

Since the functional $\mathscr{J}_{\rho}$ is proper, convex, and lower semicontinuous, it follows that its subdifferential $\partial \mathscr{F}_{\rho}$ is a maximal monotone operator.

In the following two lemmas, we establish a relation between the null space of the operator $A_{\rho}:=\partial \mathscr{J}_{\rho}$ and its range.

Lemma 18. Let $\mathcal{N}\left(A_{\rho}\right)$ denote the null space of the operator $A_{\rho}$. Then

$$
\mathscr{N}\left(A_{\rho}\right)=C \mathbf{1}=\{C=(c, c): c \in \mathbb{R}\}
$$

that is, $\mathcal{N}\left(A_{\rho}\right)$ consists of all the real constant functions on $\bar{\Omega}$.

Proof. We say that $U \in \mathcal{N}\left(A_{\rho}\right)$ if and only if (by definition) $U=\left(u,\left.u\right|_{\partial \Omega}\right)$ is a weak solution of

$$
\begin{aligned}
& -\Delta_{p} u=0, \quad \text { in } \Omega \text {, } \\
& b(x)|\nabla u|^{p-2} \partial_{n} u-\rho b(x) \Delta_{q, \Gamma} u=0, \quad \text { on } \partial \Omega .
\end{aligned}
$$

A function $U=\left(u,\left.u\right|_{\partial \Omega}\right) \in \mathscr{V}_{\rho} \cap \mathbb{X}_{2}$ is said to be a weak solution of (70), if, for every $V=\left(v,\left.v\right|_{\partial \Omega}\right) \in \mathscr{V}_{\rho} \cap \mathbb{X}_{2}$, there holds

$$
\begin{aligned}
\mathscr{A}_{\rho}(U, V):= & \int_{\Omega}|\nabla u|^{p-2} \nabla u \cdot \nabla v d x \\
& +\rho \int_{\partial \Omega}\left|\nabla_{\Gamma} u\right|^{q-2} \nabla_{\Gamma} u \cdot \nabla_{\Gamma} v d \sigma=0 .
\end{aligned}
$$

Let $C:=(c, c)$ with $c \in \mathbb{R}$. Then it is clear that $C \in \mathcal{N}\left(A_{\rho}\right)$.

Conversely, let $U=\left(u,\left.u\right|_{\partial \Omega}\right) \in \mathcal{N}\left(A_{\rho}\right)$. Then, it follows from (71) that

$$
\mathscr{A}_{\rho}(U, U):=\int_{\Omega}|\nabla u|^{p} d x+\rho \int_{\partial \Omega}\left|\nabla_{\Gamma} u\right|^{q} d \sigma=0 .
$$

Since $\Omega$ is bounded and connected, this implies that $u$ is equal to a constant. Therefore, $U=C 1$ and this completes the proof.

Lemma 19. The range of the operator $A_{\rho}$ is given by

$$
\begin{aligned}
& \mathscr{R}\left(A_{\rho}\right)=\left\{F:=(f, g) \in \mathbb{X}_{2}: \int_{\bar{\Omega}} F d \mu:=\int_{\Omega} f d x\right. \\
& \left.\quad+\int_{\partial \Omega} g \frac{d \sigma}{b(x)}=0\right\} .
\end{aligned}
$$

Proof. Let $F \in \mathscr{R}\left(A_{\rho}\right) \subset \mathbb{X}_{2}$. Then there exists $U=$ $\left(u,\left.u\right|_{\partial \Omega}\right) \in D\left(A_{\rho}\right)$ such that $A_{\rho}(U)=F$. More precisely, for every $V=\left(v,\left.v\right|_{\partial \Omega}\right) \in \mathscr{V}_{\rho} \cap \mathbb{X}_{2}$, we have

$$
\begin{aligned}
\mathscr{A}_{\rho}(U, V)= & \int_{\Omega}|\nabla u|^{p-2} \nabla u \cdot \nabla v d x \\
& +\rho \int_{\partial \Omega}\left|\nabla_{\Gamma} u\right|^{q-2} \nabla_{\Gamma} u \cdot \nabla_{\Gamma} v d \sigma \\
= & \int_{\bar{\Omega}} F V d \mu .
\end{aligned}
$$

Taking $V=(1,1) \in \mathscr{V}_{\rho} \cap \mathbb{X}_{2}$, we obtain that $\int_{\bar{\Omega}} F d \mu=0$. Hence

$$
\mathscr{R}\left(A_{\rho}\right) \subseteq\left\{F \in \mathbb{X}_{2}: \int_{\bar{\Omega}} F d \mu=0\right\}
$$

Let us now prove the converse. To this end, let $F \in \mathbb{X}_{2}$ be such that $\int_{\bar{\Omega}} F d \mu=0$. We have to show that $F \in \mathscr{R}\left(A_{\rho}\right)$; that is, there exists $U \in \mathscr{V}_{\rho} \cap \mathbb{X}_{2}$ such that (73) holds, for every $V \in \mathscr{V}_{\rho} \cap \mathbb{X}_{2}$. To this end, consider

$$
\begin{gathered}
\mathscr{V}_{\rho, 0}:=\left\{U=\left(u,\left.u\right|_{\partial \Omega}\right) \in \mathscr{V}_{\rho} \cap \mathbb{X}_{2}: \int_{\bar{\Omega}} U d \mu\right. \\
\left.:=\int_{\Omega} u d x+\int_{\partial \Omega} u \frac{d \sigma}{b}=0\right\} .
\end{gathered}
$$

It is clear that $\mathscr{V}_{\rho, 0}$ is a closed linear subspace of $\mathscr{V}_{\rho} \cap$ $\mathbb{X}_{2} \hookrightarrow \mathbb{X}_{2}$ and therefore is a reflexive Banach space. Using [23, Section 1.1], we have that the norm

$$
\|U\|_{\mathscr{V}_{\rho, 0}}:=\|\nabla u\|_{p, \Omega}+\rho\left\|\nabla_{\Gamma} u\right\|_{q, \partial \Omega}
$$

defines an equivalent norm on $\mathscr{V}_{\rho, 0}$. Hence, there exists a constant $C>0$ such that for every $U \in \mathscr{V}_{\rho, 0}$

$$
\|\mid U\|_{2} \leq C\|U\|_{\mathscr{V}_{\rho, 0}}:=\|\nabla u\|_{p, \Omega}+\rho\left\|\nabla_{\Gamma} u\right\|_{q, \partial \Omega} .
$$

Define the functional $\mathscr{F}_{\rho}: \mathscr{V}_{\rho, 0} \rightarrow \mathbb{R}$ by

$$
\begin{aligned}
\mathscr{F}_{\rho}(U)= & \frac{1}{p} \int_{\Omega}|\nabla u|^{p} d x+\frac{\rho}{q} \int_{\partial \Omega}\left|\nabla_{\Gamma} u\right|^{q} d \sigma \\
& -\int_{\bar{\Omega}} F U d \mu .
\end{aligned}
$$

It is easy to see that $\mathscr{F}_{\rho}$ is convex and lower semicontinuous on $\mathbb{X}_{2}$ (see Proposition 16). We show now that $\mathscr{F}_{\rho}$ is coercive. By exploiting a classical Hölder inequality and using (78), we have

$$
\begin{aligned}
\left|\int_{\bar{\Omega}} F U d \mu\right| & \leq\left.\left. C\|F\|\right|_{2}\left\|\left|U\left\|_{2} \leq C\right\|\right| F\right\|\right|_{2}\|U\|_{\mathscr{V}_{\rho, 0}} \\
& =C\left\||F \||_{2}\left(\|\nabla u\|_{p, \Omega}+\rho\left\|\nabla_{\Gamma} u\right\|_{q, \partial \Omega}\right) .\right.
\end{aligned}
$$

Obviously, this estimate yields

$$
-\int_{\bar{\Omega}} F U d \mu \geq-C\|\mid F\|_{2}\left(\|\nabla u\|_{p, \Omega}+\rho\left\|\nabla_{\Gamma} u\right\|_{q, \partial \Omega}\right) .
$$


Therefore, from (81), we immediately get

$$
\begin{aligned}
\frac{\mathscr{F}_{\rho}(U)}{\|U\|_{\mathscr{V}_{\rho, 0}}} \geq & \frac{(1 / p)\|\nabla u\|_{p, \Omega}^{p}+(\rho / q)\left\|\nabla_{\Gamma} u\right\|_{q, \partial \Omega}^{q}}{\|\nabla u\|_{p, \Omega}+\rho\left\|\nabla_{\Gamma} u\right\|_{q, \partial \Omega}} \\
& -C\|\mid F\|_{2} .
\end{aligned}
$$

This inequality implies that

$$
\lim _{\|U\|_{\mathscr{V}_{\rho, 0} \rightarrow+\infty}} \frac{\mathscr{F}_{\rho}(U)}{\|U\|_{\mathscr{V}_{\rho, 0}}}=+\infty,
$$

and this shows that the functional $\mathscr{F}_{\rho}$ is coercive. Since $\mathscr{F}_{\rho}$ is also convex and lower semicontinuous, it follows from [24, Theorem 3.3.4] that there exists a function $U^{*} \in \mathscr{V}_{\rho, 0}$ which minimizes $\mathscr{F}_{\rho}$. More precisely, for all $V \in \mathscr{V}_{\rho, 0}, \mathscr{F}_{\rho}\left(U^{*}\right) \leq$ $\mathscr{F}_{\rho}(V)$; this implies that for every $0<t \leq 1$ and every $V \in$ $\mathscr{V}_{\rho, 0}^{\rho}$

$$
\mathscr{F}_{\rho}\left(U^{*}+t V\right)-\mathscr{F}_{\rho}\left(U^{*}\right) \geq 0 .
$$

Hence

$$
\lim _{t \downarrow 0} \frac{\mathscr{F}_{\rho}\left(U^{*}+t V\right)-\mathscr{F}_{\rho}\left(U^{*}\right)}{t} \geq 0 .
$$

Using the Lebesgue Dominated Convergence, easy computation shows that

$$
\begin{aligned}
0 \leq & \lim _{t \downarrow 0} \frac{\mathscr{F}_{\rho}\left(U^{*}+t V\right)-\mathscr{F}_{\rho}\left(U^{*}\right)}{t} \\
= & \int_{\Omega}\left|\nabla u^{*}\right|^{p-2} \nabla u^{*} \cdot \nabla v d x \\
& +\rho \int_{\partial \Omega}\left|\nabla_{\Gamma} u^{*}\right|^{q-2} \nabla_{\Gamma} u^{*} \cdot \nabla_{\Gamma} v d \sigma-\int_{\bar{\Omega}} F V d \mu .
\end{aligned}
$$

Changing $V$ to $-V$ in (86) gives that

$$
\begin{aligned}
& \int_{\Omega}\left|\nabla u^{*}\right|^{p-2} \nabla u^{*} \cdot \nabla v d x \\
& \quad+\rho \int_{\partial \Omega}\left|\nabla_{\Gamma} u^{*}\right|^{q-2} \nabla_{\Gamma} u^{*} \cdot \nabla_{\Gamma} v d \sigma=\int_{\bar{\Omega}} F V d \mu,
\end{aligned}
$$

for every $V \in \mathscr{V}_{\rho, 0}$. Now, let $V \in \mathscr{V}_{\rho} \cap \mathbb{X}_{2}$. Writing $V=$ $V-C+C$ with $C=(c, c)$

$$
c:=\frac{1}{\left(\lambda_{1}+\lambda_{2}\right)}\left(\int_{\Omega} v d x+\int_{\partial \Omega} v \frac{d \sigma}{b}\right),
$$

and using the fact that $\int_{\bar{\Omega}} F d \mu=0$, we obtain, for every $V \in$ $\mathscr{V}_{\rho} \cap \mathbb{X}_{2}$, that

$$
\begin{aligned}
& \int_{\Omega}\left|\nabla u^{*}\right|^{p-2} \nabla u^{*} \cdot \nabla v d x \\
& \quad+\rho \int_{\partial \Omega}\left|\nabla_{\Gamma} u^{*}\right|^{q-2} \nabla_{\Gamma} u^{*} \cdot \nabla_{\Gamma} v d \sigma=\int_{\bar{\Omega}} F V d \mu .
\end{aligned}
$$

Therefore, $A_{\rho}(U)=F$. Hence, $F \in \mathscr{R}\left(A_{\rho}\right)$ and this completes the proof of the lemma.
The following result is a direct consequence of Lemmas 18 and 19. This is the main result of this section.

Theorem 20. The operator $A_{\rho}=\partial \mathscr{J}_{\rho}$ satisfies the following type of "quasilinear" Fredholm alternative:

$$
\mathscr{R}\left(A_{\rho}\right)=\mathscr{N}\left(A_{\rho}\right)^{\perp}=\left\{F \in \mathbb{X}_{2}:\langle F, \mathbf{1}\rangle_{\mathbb{X}_{2}}=0\right\}
$$

\section{Necessary and Sufficient Conditions for Existence of Solutions}

In this section, we prove the first main result (cf. Theorem 1) for problem (1). Before we do so, we will need the following results from maximal monotone operators theory and convex analysis.

Definition 21. Let $\mathscr{H}$ be a real Hilbert space. Two subsets $K_{1}$ and $K_{2}$ of $\mathscr{H}$ are said to be almost equal, written as $K_{1} \simeq K_{2}$, if $K_{1}$ and $K_{2}$ have the same closure and the same interior, that is, $\overline{K_{1}}=\overline{K_{2}}$ and $\operatorname{int}\left(K_{1}\right)=\operatorname{int}\left(K_{2}\right)$.

The following abstract result is taken from [9, Theorem 3 and Generalization in p. 173-174].

Theorem 22 (Brézis-Haraux). Let $A$ and $B$ be subdifferentials of proper convex lower semicontinuous functionals $\varphi_{1}$ and $\varphi_{2}$, respectively, on a real Hilbert space $\mathscr{H}$ with $D\left(\varphi_{1}\right) \cap D\left(\varphi_{2}\right) \neq$ $\emptyset$, and let $C$ be the subdifferential of the proper, convex lower semicontinuous functional $\varphi_{1}+\varphi_{2}$; that is, $C=\partial\left(\varphi_{1}+\varphi_{2}\right)$. Then

$$
\begin{array}{r}
\mathscr{R}(A)+\mathscr{R}(B) \subset \overline{\mathscr{R}(C)}, \\
\operatorname{int}(\mathscr{R}(A)+\mathscr{R}(B)) \subset \mathscr{R}(C) .
\end{array}
$$

In particular, if the operator $A+B$ is maximal monotone, then

$$
\mathscr{R}(A+B) \simeq \mathscr{R}(A)+\mathscr{R}(B),
$$

and this is the case if $\partial\left(\varphi_{1}+\varphi_{2}\right)=\partial \varphi_{1}+\partial \varphi_{2}$.

4.1. Assumptions and Intermediate Results. Let us recall that the aim of this section is to establish some necessary and sufficient conditions for the solvability of the following nonlinear elliptic problem:

$$
\begin{aligned}
-\Delta_{p} u+\alpha_{1}(u) & =f, \quad \text { in } \Omega, \\
b(x)|\nabla u|^{p-2} \partial_{n} u-\rho b(x) \Delta_{q, \Gamma} u+\alpha_{2}(u) & =g,
\end{aligned}
$$

on $\partial \Omega$,

where $p, q \in(1,+\infty)$ are fixed. We also assume that $\alpha_{j}: \mathbb{R} \rightarrow$ $\mathbb{R}(j=1,2)$ satisfy the following assumptions.

Assumption 23. The functions $\alpha_{j}: \mathbb{R} \rightarrow \mathbb{R}(j=1,2)$ are odd, monotone nondecreasing, and continuous and satisfy $\alpha_{j}(0)=$ 0 . 
Let $\widetilde{\alpha}_{j}$ be the inverse of $\alpha_{j}$. We define the functions $\Lambda_{j}, \widetilde{\Lambda}_{j}: \mathbb{R} \rightarrow \mathbb{R}_{+}(j=1,2)$ by

$$
\begin{aligned}
& \Lambda_{j}(t):=\int_{0}^{|t|} \alpha_{j}(s) d s, \\
& \widetilde{\Lambda}_{j}(t):=\int_{0}^{|t|} \widetilde{\alpha}_{j}(s) d s .
\end{aligned}
$$

Then it is clear that $\Lambda_{j}, \widetilde{\Lambda}_{j}$ are even, convex, and monotone increasing on $\mathbb{R}_{+}$, with $\Lambda_{j}(0)=\widetilde{\Lambda}_{j}(0)$, for each $j=1,2$. Moreover, since $\alpha_{j}$ are odd, we have $\Lambda_{j}^{\prime}(t)=\alpha_{j}(t)$, for all $t \in \mathbb{R}$ and $j=1,2$, with a similar relation holding for $\widetilde{\Lambda}_{j}$ as well. The following result whose proof is included in [19, Chap. I, Section 1.3, Theorem 3] holds.

Lemma 24. The functions $\Lambda_{j}$ and $\widetilde{\Lambda}_{j}(j=1,2)$ satisfy (43) and (44). More precisely, for all $s, t \in \mathbb{R}$

$$
s t \leq \Lambda_{j}(s)+\widetilde{\Lambda}_{j}(t) .
$$

If $s=\alpha_{j}(t)$ or $t=\widetilde{\alpha}_{j}(s)$, then we also have equality; that is,

$$
\widetilde{\Lambda}_{j}\left(\alpha_{j}(s)\right)=s \alpha_{j}(s)-\Lambda_{j}(s), \quad j=1,2 .
$$

We note that, in [19], the statement of Lemma 24 assumed that $\Lambda_{j}, \widetilde{\Lambda}_{j}$ are $\mathcal{N}$-functions in the sense of Definition 7 . However, the conclusion of that result holds under the weaker hypotheses of Lemma 24.

Define the functional $\mathscr{F}_{2}: \mathbb{X}_{2} \rightarrow[0,+\infty]$ by

$$
\begin{aligned}
& \mathscr{F}_{2}(u, v) \\
& := \begin{cases}\int_{\Omega} \Lambda_{1}(u) d x+\int_{\partial \Omega} \Lambda_{2}(v) \frac{d \sigma}{b}, & \text { if }(u, v) \in D\left(\mathscr{F}_{2}\right), \\
+\infty, & \text { if }(u, v) \in \mathbb{X}_{2} \backslash D\left(\mathscr{F}_{2}\right),\end{cases}
\end{aligned}
$$

with the effective domain

$$
\begin{aligned}
& D\left(\mathscr{J}_{2}\right):=\left\{(u, v) \in \mathbb{X}_{2}: \int_{\Omega} \Lambda_{1}(u) d x\right. \\
& \left.+\int_{\partial \Omega} \Lambda_{2}(v) \frac{d \sigma}{b}<\infty\right\} .
\end{aligned}
$$

Lemma 25. Let $\alpha_{j}(j=1,2)$ satisfy Assumption 23. Then the functional $\mathscr{F}_{2}$ is proper, convex, and lower semicontinuous on $\mathbb{X}_{2}$.

Proof. It is routine to check that $\mathscr{F}_{2}$ is convex and proper. This follows easily from the convexity of $\Lambda_{j}$ and the fact that $\Lambda_{j}(0)=0$. To show the lower semicontinuity on $\mathbb{X}_{2}$, let $U_{n}=\left(u_{n}, v_{n}\right) \in D\left(\mathscr{g}_{2}\right)$ be such that $U_{n} \rightarrow U:=(u, v)$ in $\mathbb{X}_{2}$ and $\mathscr{J}_{2}\left(U_{n}\right) \leq C$ for some constant $C>0$. Since $U_{n} \rightarrow U$ in $\mathbb{X}_{2}$, then there is a subsequence, which we also denote by $U_{n}=\left(u_{n}, v_{n}\right)$, such that $u_{n} \rightarrow u$ a.e. on $\Omega$ and $v_{n} \rightarrow v \sigma$, a.e. on $\Gamma$. Since $\Lambda_{j}(\cdot)$ are continuous (thus, lower semicontinuous), we have

$$
\begin{aligned}
& \Lambda_{1}(u) \leq \liminf _{n \rightarrow \infty} \Lambda_{1}\left(u_{n}\right), \\
& \Lambda_{2}(v) \leq \liminf _{n \rightarrow \infty} \Lambda_{2}\left(v_{n}\right) .
\end{aligned}
$$

By Fatou's Lemma, we obtain

$$
\begin{aligned}
\int_{\Omega} \Lambda_{1}(u) d x & \leq \int_{\Omega} \liminf _{n \rightarrow \infty} \Lambda_{1}\left(u_{n}\right) d x \\
& \leq \liminf _{n \rightarrow \infty} \int_{\Omega} \Lambda_{1}\left(u_{n}\right) d x, \\
\int_{\partial \Omega} \Lambda_{2}(v) \frac{d \sigma}{b} & \leq \int_{\partial \Omega} \liminf _{n \rightarrow \infty} \Lambda_{2}\left(v_{n}\right) \frac{d \sigma}{b} \\
& \leq \liminf _{n \rightarrow \infty} \int_{\partial \Omega} \Lambda_{2}\left(v_{n}\right) \frac{d \sigma}{b} .
\end{aligned}
$$

Hence, $\mathscr{J}_{2}$ is lower semicontinuous on $\mathbb{X}_{2}$.

We have the following result whose proof is contained in [19, Chap. III, Section 3.1, Theorem 2].

Lemma 26. Let $\alpha_{j}(j=1,2)$ satisfy Assumption 23 and assume that there exist constants $C_{j}>1(j=1,2)$ such that

$$
\Lambda_{j}(2 t) \leq C_{j} \Lambda_{j}(t), \quad \forall t \in \mathbb{R} .
$$

Then $D\left(\mathscr{J}_{2}\right)$ is a vector space.

Let the operator $B_{2}$ be defined by

$$
\begin{aligned}
& D\left(B_{2}\right)=\left\{U:=(u, v) \in \mathbb{X}_{2}:\left(\alpha_{1}(u), \alpha_{2}(v)\right) \in \mathbb{X}_{2}\right\}, \\
& B_{2}(U)=\left(\alpha_{1}(u), \alpha_{2}(v)\right) .
\end{aligned}
$$

We have the following result.

Lemma 27. Let the assumptions of Lemma 26 be satisfied. Then the subdifferential of $\mathscr{F}_{2}$ and the operator $B_{2}$ coincide; that is, for all $(u, v) \in D\left(B_{2}\right)=D\left(\partial \mathscr{F}_{2}\right)$

$$
\partial \mathscr{F}_{2}(u, v)=B_{2}(u, v) .
$$

Proof. Let $U=(u, v) \in D\left(\mathscr{F}_{2}\right)$ and $F=(f, g) \in \partial \mathscr{F}_{2}(u, v)$. Then, by definition, $F \in \mathbb{X}_{2}$ and, for every $V=\left(u_{1}, v_{1}\right) \in$ $D\left(\mathscr{F}_{2}\right)$, we get

$$
\int_{\bar{\Omega}} F(V-U) d \mu \leq \mathscr{F}_{2}(V)-\mathscr{F}_{2}(U) .
$$

Let $V=U+t W$, with $W=\left(u_{2}, v_{2}\right) \in D\left(\mathscr{F}_{2}\right)$ and $0<t \leq 1$. Then by Lemma $26, V=U+t W \in D\left(\mathscr{F}_{2}\right)$. Now, dividing by $t$ and taking the limit as $t \downarrow 0$, we obtain

$$
\int_{\Omega} F W d \mu \leq \int_{\Omega} \alpha_{1}(u) u_{2} d x+\int_{\partial \Omega} \alpha_{2}(v) v_{2} \frac{d \sigma}{b} .
$$

Changing $W$ to $-W$ in (105) gives that

$$
\int_{\bar{\Omega}} F W d \mu=\int_{\Omega} \alpha_{1}(u) u_{2} d x+\int_{\partial \Omega} \alpha_{2}(v) v_{2} \frac{d \sigma}{b} .
$$

In particular, if $W=\left(u_{2}, 0\right)$ with $u_{2} \in \mathscr{D}(\Omega)$, we have

$$
\int_{\Omega} f u_{2} d x=\int_{\Omega} \alpha_{1}(u) u_{2} d x
$$


and this shows that $\alpha_{1}(u)=f$. Similarly, one obtains that $\alpha_{2}(v)=g$. We have shown that $U \in D\left(B_{2}\right)$ and

$$
B_{2}(U):=B_{2}(u, v)=\left(\alpha_{1}(u), \alpha_{2}(v)\right)=(f, g) .
$$

Conversely, let $U=(u, v) \in D\left(B_{2}\right)$ and set $F=(f, g):=$ $B_{2}(u, v)=\left(\alpha_{1}(u), \alpha_{2}(v)\right)$. Since $\left(\alpha_{1}(u), \alpha_{2}(v)\right) \in \mathbb{X}_{2}$, from (94) and (101), it follows that

$$
\int_{\Omega} \Lambda_{1}(u) d x+\int_{\partial \Omega} \Lambda_{2}(v) \frac{d \sigma}{b}<\infty
$$

Hence, $U=(u, v) \in D\left(\mathscr{J}_{2}\right)$. Let $V=\left(u_{1}, v_{1}\right) \in D\left(\mathscr{J}_{2}\right)$. Using Lemma 24, we obtain

$$
\begin{aligned}
\alpha_{1}(u)\left(u_{1}-u\right) & =\alpha_{1}(u) u_{1}-\alpha_{1}(u) u \\
& \leq \Lambda_{1}\left(u_{1}\right)+\Lambda_{1}\left(\alpha_{1}(u)\right)-\alpha_{1}(u) u \\
& =\Lambda_{1}\left(u_{1}\right)-\Lambda_{1}(u)
\end{aligned}
$$

and similarly

$$
\alpha_{2}(v)\left(v_{1}-v\right) \leq \Lambda_{2}\left(v_{1}\right)-\Lambda_{2}(v) .
$$

Therefore

$$
\begin{aligned}
\int_{\bar{\Omega}} F(V-U) d \mu= & \int_{\Omega} \alpha_{1}(u)\left(u_{1}-u\right) d x \\
& +\int_{\partial \Omega} \alpha_{2}(v)\left(v_{1}-v\right) \frac{d \sigma}{b} \\
& \leq \mathscr{J}_{2}(V)-\mathscr{J}_{2}(U) .
\end{aligned}
$$

By definition, this shows that $F=\left(\alpha_{1}(u), \alpha_{2}(v)\right)=B_{2}(U) \epsilon$ $\partial \mathscr{F}_{2}(U)$. We have shown that $U \in D\left(\partial \mathscr{F}_{2}\right)$ and $B_{2}(U) \in$ $\partial \mathscr{F}_{2}(U)$. This completes the proof of the lemma.

Next, we define the functional $\mathscr{J}_{3, \rho}: \mathbb{X}_{2} \rightarrow[0,+\infty]$ by

$$
\begin{aligned}
& \mathscr{J}_{3, \rho}(U) \\
& = \begin{cases}\mathscr{F}_{\rho}(U)+\mathscr{F}_{2}(U) & \text { if } U \in D\left(\mathscr{J}_{3, \rho}\right):=D\left(\mathscr{F}_{\rho}\right) \cap D\left(\mathscr{F}_{2}\right), \\
+\infty & \text { if } U \in \mathbb{X}_{2} \backslash D\left(\mathscr{F}_{3, \rho}\right) .\end{cases}
\end{aligned}
$$

Note that for $\rho=0$

$$
\begin{aligned}
& D\left(\mathscr{J}_{3,0}\right)=\left\{U=\left(u,\left.u\right|_{\partial \Omega}\right) \in D\left(\mathscr{J}_{2}\right): u \in W^{1, p}(\Omega)\right. \\
& \left.\cap L^{2}(\Omega),\left.u\right|_{\partial \Omega} \in L^{2}(\partial \Omega)\right\},
\end{aligned}
$$

while for $\rho=1$

$$
\begin{gathered}
D\left(\mathscr{J}_{3,1}\right)=\left\{U=\left(u,\left.u\right|_{\partial \Omega}\right) \in D\left(\mathscr{J}_{2}\right): u \in W^{1, p}(\Omega)\right. \\
\left.\cap L^{2}(\Omega),\left.u\right|_{\partial \Omega} \in W^{1, q}(\partial \Omega) \cap L^{2}(\partial \Omega)\right\} .
\end{gathered}
$$

We have the following result.
Lemma 28. Let the assumptions of Lemma 26 be satisfied. Then the subdifferential of the functional $\mathcal{F}_{3, \rho}$ is given by

$$
\begin{aligned}
& D\left(\partial_{3, \rho}\right)=\left\{U=\left(u, u_{\mid \partial \Omega}\right) \in D\left(\mathscr{J}_{3, \rho}\right):-\Delta_{p} u\right. \\
& +\alpha_{1}(u) \in L^{2}(\Omega), b(x)|\nabla u|^{p-2} \partial_{n} u \\
& \left.\quad-b(x) \rho \Delta_{q, \Gamma} u+\alpha_{2}(u) \in L^{2}\left(\partial \Omega, \frac{d \sigma}{b}\right)\right\}, \\
& \partial \mathscr{F}_{3, \rho}(U)=\left(-\Delta_{p} u+\alpha_{1}(u), b(x)|\nabla u|^{p-2} \partial_{n} u\right. \\
& \left.\quad-b(x) \rho \Delta_{q, \Gamma} u+\alpha_{2}(u)\right) .
\end{aligned}
$$

In particular, if, for every $U=\left(u, u_{\mid \partial \Omega}\right) \in D\left(\mathscr{J}_{3, \rho}\right)$, the function $\left(\alpha_{1}(u), \alpha_{2}(u)\right) \in \mathbb{X}_{2}$, then

$$
\partial \mathscr{F}_{3, \rho}:=\partial\left(\mathscr{J}_{\rho}+\mathscr{J}_{2}\right)=\partial \mathscr{J}_{\rho}+\partial \mathscr{J}_{2} .
$$

Proof. We calculate the subdifferential $\partial_{3, \rho}$. Let $F=(f, g) \epsilon$ $\partial \mathscr{J}_{3, \rho}(U)$; that is, $F \in \mathbb{X}_{2}, U \in D\left(\mathscr{J}_{3, \rho}\right)=D\left(\mathscr{J}_{\rho}\right) \cap D\left(\mathscr{F}_{2}\right)$, and for every $V \in D\left(\mathscr{J}_{3, \rho}\right)$, we have

$$
\int_{\bar{\Omega}} F(V-U) d \mu \leq \mathscr{J}_{3, \rho}(V)-\mathscr{F}_{3, \rho}(U) .
$$

Proceeding as in Remark 17 and the proof of Lemma 27, we obtain that

$$
\begin{gathered}
-\Delta_{p} u+\alpha_{1}(u)=f \\
\text { in } \mathscr{D}(\Omega)^{\prime}, \\
b(x)|\nabla u|^{p-2} \partial_{n} u-b(x) \rho \Delta_{q, \Gamma} u+\alpha_{2}(u)=g \\
\text { weakly on } \partial \Omega .
\end{gathered}
$$

Noting that $\partial \mathscr{J}_{3, \rho}$ is also a single-valued operator (which follows from the assumptions on $\alpha_{j}$ and $\Lambda_{j}$ ), we easily obtain (116), and this completes the proof of the first part.

To show the last part, note that it is clear that $\partial \mathscr{J}_{\rho}+\partial \mathscr{F}_{2} \mathrm{C}$ $\partial \mathscr{F}_{3, \rho}$ always holds. To show the converse inclusion, let us assume that, for every $U=\left(u, u_{\mid \partial \Omega}\right) \in D\left(\mathscr{J}_{3, \rho}\right)$, the function $\left(\alpha_{1}(u), \alpha_{2}(u)\right) \in \mathbb{X}_{2}$. Then it follows from (68), (102) (since $\left.\partial \mathscr{F}_{2}=B_{2}\right)$, and (116) that $D\left(\partial \mathscr{F}_{3, \rho}\right)=D\left(\partial \mathscr{F}_{\rho}\right) \cap D\left(\partial \mathscr{F}_{2}\right)$ and

$$
\begin{aligned}
& \partial \mathscr{J}_{3, \rho}(U)=\left(-\Delta_{p} u+\alpha_{1}(u), b(x)|\nabla u|^{p-2} \partial_{n} u\right. \\
& \left.\quad-b(x) \rho \Delta_{q, \Gamma} u+\alpha_{2}(u)\right) \\
& \quad=\left(-\Delta_{p} u, b(x)|\nabla u|^{p-2} \partial_{n} u-b(x) \rho \Delta_{q, \Gamma} u\right) \\
& \quad+\left(\alpha_{1}(u), \alpha_{2}(u)\right)=\partial \mathscr{F}_{\rho}(U)+\partial \mathscr{J}_{2}(U) .
\end{aligned}
$$

This completes the proof.

The following lemma is the main ingredient in the proof of Theorem 31 below. 
Lemma 29. Let $B_{1}:=A_{\rho}$ and set $B_{3}:=\partial \mathscr{J}_{3, \rho}$. Then

$$
\begin{array}{r}
\mathscr{R}\left(B_{1}\right)+\mathscr{R}\left(B_{2}\right) \subset \overline{\mathscr{R}\left(B_{3}\right)}, \\
\operatorname{int}\left(\mathscr{R}\left(B_{1}\right)+\mathscr{R}\left(B_{2}\right)\right) \subset \mathscr{R}\left(B_{3}\right) .
\end{array}
$$

In particular, if, for every $U=\left(u, u_{\mid \partial \Omega}\right) \in D\left(\mathscr{J}_{3, \rho}\right)$, the function $\left(\alpha_{1}(u), \alpha_{2}(u)\right) \in \mathbb{X}_{2}$, then

$$
\mathscr{R}\left(B_{3}\right):=\mathscr{R}\left(B_{1}+B_{2}\right) \simeq \mathscr{R}\left(B_{1}\right)+\mathscr{R}\left(B_{2}\right) .
$$

Proof. By Remark 17 and Lemmas 27 and 28, the operators $B_{1}, B_{2}$, and $B_{3}$ are subdifferentials of proper, convex, and lower semicontinuous functionals $\mathscr{J}_{\rho}, \mathscr{J}_{2}$, and $\mathscr{J}_{\rho}+\mathscr{J}_{2}$, respectively, on $\mathbb{X}_{2}$. Hence, $B_{1}, B_{2}$, and $B_{3}$ are maximal monotone operators. In particular, if $\left(\alpha_{1}(u), \alpha_{2}(u)\right) \in \mathbb{X}_{2}$, for every $U=\left(u, u_{\mid \partial \Omega}\right) \in D\left(\mathscr{J}_{3, \rho}\right)$, then, by Lemma 28 , one has $B_{3}=B_{1}+B_{2}$. Now, the lemma follows from the celebrated Brézis-Haraux result in Theorem 22.

4.2. Statement and Proof of the Main Result. Next, let $\mathscr{V}_{\rho}:=$ $D\left(\mathscr{J}_{3, \rho}\right)$ be given by (114) if $\rho=0$ and by (115) if $\rho=1$.

Definition 30. Let $F=(f, g) \in \mathbb{X}_{2}$. A function $u \in W^{1, p}(\Omega)$ is said to be a weak solution of (93), if $\alpha_{1}(u) \in L^{1}(\Omega), \alpha_{2}(u) \in$ $L^{1}(\partial \Omega),\left.u\right|_{\partial \Omega} \in W^{1, q}(\partial \Omega)$, if $\rho>0$, and

$$
\begin{gathered}
\int_{\Omega}|\nabla u|^{p-2} \nabla u \cdot \nabla v d x+\rho \int_{\partial \Omega}\left|\nabla_{\Gamma} u\right|^{q-2} \nabla_{\Gamma} u \cdot \nabla_{\Gamma} v d \sigma \\
\quad+\int_{\Omega} \alpha_{1}(u) v d x+\int_{\partial \Omega} \alpha_{2}(u) v \frac{d \sigma}{b} \\
=\int_{\Omega} f v d x+\int_{\partial \Omega} g v \frac{d \sigma}{b},
\end{gathered}
$$

for every $v \in W^{1, p}(\Omega) \cap C(\bar{\Omega})$ with $\left.v\right|_{\partial \Omega} \in W^{1, q}(\partial \Omega)$, if $\rho>0$.

Recall that $\lambda_{1}:=\int_{\Omega} d x$ and $\lambda_{2}:=\int_{\partial \Omega}(d \sigma / b)$. We also define the average $\langle F\rangle_{\bar{\Omega}}$ of $F=(f, g)$ with respect to the measure $\mu$, as follows:

$$
\begin{aligned}
\langle F\rangle_{\bar{\Omega}} & :=\frac{1}{\mu(\bar{\Omega})} \int_{\bar{\Omega}} F d \mu \\
& =\frac{1}{\mu(\bar{\Omega})}\left(\int_{\Omega} f d x+\int_{\partial \Omega} g \frac{d \sigma}{b}\right),
\end{aligned}
$$

where $\mu(\bar{\Omega})=\lambda_{1}+\lambda_{2}$. Now, we are ready to state the main result of this section.

Theorem 31. Let $\alpha_{j}(j=1,2)$ satisfy Assumption 23 and assume that the functions $\Lambda_{j}(j=1,2)$ satisfy (101). Let $F=$ $(f, g) \in \mathbb{X}_{2}$. The following hold:

(a) Suppose that the nonlinear elliptic problem (93) possesses a weak solution. Then

$$
\langle F\rangle_{\Omega} \in \frac{\lambda_{1} \mathscr{R}\left(\alpha_{1}\right)+\lambda_{2} \mathscr{R}\left(\alpha_{2}\right)}{\lambda_{1}+\lambda_{2}} .
$$

(b) Assume that

$$
\langle F\rangle_{\bar{\Omega}} \in \operatorname{int}\left(\frac{\lambda_{1} \mathscr{R}\left(\alpha_{1}\right)+\lambda_{2} \mathscr{R}\left(\alpha_{2}\right)}{\lambda_{1}+\lambda_{2}}\right) .
$$

Then the nonlinear elliptic problem (93) has at least one weak solution.

Proof. We show that condition (125) is necessary. Let $F$ := $(f, g) \in \mathbb{X}_{2}$ and let $U=\left(u, u_{\mid \partial \Omega}\right) \in D\left(B_{3}\right) \subset \mathscr{V}_{\rho}$ be a weak solution of $B_{3} U=F$. Then, by definition, for every $V=\left(v,\left.v\right|_{\partial \Omega}\right) \in \mathscr{V}_{\rho},(123)$ holds. Taking $v \equiv 1$ in (123) yields

$$
\int_{\Omega} f d x+\int_{\partial \Omega} g \frac{d \sigma}{b}=\int_{\Omega} \alpha_{1}(u) d x+\int_{\partial \Omega} \alpha_{2}(u) \frac{d \sigma}{b} .
$$

Hence

$$
\int_{\Omega} f d x+\int_{\partial \Omega} g \frac{d \sigma}{b} \in\left(\lambda_{1} \mathscr{R}\left(\alpha_{1}\right)+\lambda_{2} \mathscr{R}\left(\alpha_{2}\right)\right),
$$

and so (125) holds. This completes the proof of part (a).

We show that condition (126) is sufficient.

(i) First, let $C \in \mathbf{C}$, where

$$
\mathrm{C}:=\left\{C=\left(c_{1}, c_{2}\right):\left(c_{1}, c_{2}\right) \in \mathscr{R}\left(\alpha_{1}\right) \times \mathscr{R}\left(\alpha_{2}\right)\right\} .
$$

By definition, one has that $\mathbf{C} \subset \mathscr{R}\left(B_{2}\right)$ since $c_{1}=\alpha_{1}\left(d_{1}\right)$ for some constant function $d_{1}$ on $\Omega$ and $c_{2}=\alpha_{2}\left(d_{2}\right)$ for some constant function $d_{2}$ on $\partial \Omega$. Let $F \in \mathbb{X}_{2}$ be such that (126) holds. We must show $F \in \mathscr{R}\left(B_{3}\right)$. By (126), we may choose $C=\left(c_{1}, c_{2}\right) \in \mathbf{C}$ such that

$$
\begin{aligned}
\langle F\rangle_{\bar{\Omega}} & =\frac{\lambda_{1} c_{1}+\lambda_{2} c_{2}}{\lambda_{1}+\lambda_{2}} \\
& \in \operatorname{int}\left(\frac{\lambda_{1} \mathscr{R}\left(\alpha_{1}\right)+\lambda_{2} \mathscr{R}\left(\alpha_{2}\right)}{\lambda_{1}+\lambda_{2}}\right) .
\end{aligned}
$$

Then, for $F \in \mathbb{X}_{2}$, we have $F=F_{1}+F_{2}$ with

$$
\begin{aligned}
& F_{1}:=F-C, \\
& F_{2}=C .
\end{aligned}
$$

First, $F_{1} \in \mathscr{R}\left(B_{1}\right)=\mathcal{N}\left(B_{1}\right)^{\perp}=\mathbf{1}^{\perp}$, since

$$
\begin{aligned}
\int_{\bar{\Omega}} F_{1} d \mu & =\int_{\bar{\Omega}}(F-C) d \mu \\
& =\int_{\Omega} f d x+\int_{\partial \Omega} g \frac{d \sigma}{b}-\left(\lambda_{1} c_{1}+\lambda_{2} c_{2}\right) \\
& =\left(\lambda_{1}+\lambda_{2}\right)\langle F\rangle_{\bar{\Omega}}-\left(\lambda_{1} c_{1}+\lambda_{2} c_{2}\right)=0 .
\end{aligned}
$$

Obviously, $F_{2}=C \in \mathscr{R}\left(B_{2}\right)$. Hence, it is readily seen that

$$
F \in\left(\mathscr{R}\left(B_{1}\right)+\mathscr{R}\left(B_{2}\right)\right) .
$$

(ii) Next, denote by $\mathbb{B}_{\mathbb{R}}(x, r)$ the open ball in $\mathbb{R}$ of center $x$ and radius $r>0$. Since

$$
\langle F\rangle_{\Omega} \in \operatorname{int}\left(\frac{\lambda_{1} \mathscr{R}\left(\alpha_{1}\right)+\lambda_{2} \mathscr{R}\left(\alpha_{2}\right)}{\lambda_{1}+\lambda_{2}}\right),
$$


there exists $\delta>0$ such that the open ball

$$
\mathbb{B}_{\mathbb{R}}\left(\langle F\rangle_{\bar{\Omega}}, \delta\right) \subset\left(\frac{\lambda_{1} \mathscr{R}\left(\alpha_{1}\right)+\lambda_{2} \mathscr{R}\left(\alpha_{2}\right)}{\lambda_{1}+\lambda_{2}}\right) .
$$

Since the mapping $F \mapsto\langle F\rangle_{\Omega}$ from $\mathbb{X}_{2}$ into $\mathbb{R}$ is continuous, then there exists $\varepsilon>0$ such that

$$
\langle G\rangle_{\bar{\Omega}} \in \mathbb{B}_{\mathbb{R}}\left(\langle F\rangle_{\bar{\Omega}}, \delta\right) \subset\left(\frac{\lambda_{1} \mathscr{R}\left(\alpha_{1}\right)+\lambda_{2} \mathscr{R}\left(\alpha_{2}\right)}{\lambda_{1}+\lambda_{2}}\right),
$$

for all $G \in \mathbb{X}_{2}$ satisfying $\|\mid F-G\|_{2}<\varepsilon$. It finally follows from part (i) above that $\left(\mathscr{R}\left(B_{1}\right)+\mathscr{R}\left(B_{2}\right)\right)$ contains an $\mathcal{\varepsilon}$-ball in $\mathbb{X}_{2}$ centered at $F$. Therefore

$$
F \in \operatorname{int}\left(\mathscr{R}\left(B_{1}\right)+\mathscr{R}\left(B_{2}\right)\right) \subset \mathscr{R}\left(B_{3}\right) .
$$

Consequently, problem (93) is (weakly) solvable for every function $F=(f, g) \in \mathbb{X}_{2}$, if (126) holds. This completes the proof of the theorem.

Remark 32. It is important to remark that, in order to prove Theorem 31, we do not require that $\left(\alpha_{1}(u), \alpha_{2}(u)\right)$ should belong to $\mathbb{X}_{2}$, for every $U=\left(u, u_{\mid \Gamma}\right) \in D\left(\mathscr{J}_{3, \rho}\right)$. In particular, only the assumption (121) was needed. However, if this happens, then we get the much stronger result in (122) which would require that the nonlinearities $\alpha_{1}, \alpha_{2}$ satisfy growth assumptions at infinity.

We conclude this section with the following corollary and some examples.

Corollary 33. Let the assumptions of Theorem 31 be satisfied. Let $F=(f, g) \in \mathbb{X}_{2}$. Assume that at least one of the sets $\mathscr{R}\left(\alpha_{1}\right), \mathscr{R}\left(\alpha_{2}\right)$ is open. Then the nonlinear elliptic problem (93) possesses a weak solution if and only if (126) holds.

Remark 34. Similar results to Theorem 31 and Corollary 33 were also obtained in [4, Theorem 4.4], but only when $p=$ $q=2$.

4.3. Examples. We will now give some examples as applications of Theorem 31. Let $p, q \in(1,+\infty)$ be fixed.

Example 35. Let $\alpha_{1}(s)$ or $\alpha_{2}(s)$ be equal to $\alpha(s)=c|s|^{r-1} s$, where, $r>0$. Note that $\mathscr{R}(\alpha)=\mathbb{R}$. It is easy to check that $\alpha$ satisfies all the conditions of Assumption 23 and that the function $\Lambda(t)=\int_{0}^{|t|} \alpha(s) d s$ satisfies (101). Then, it follows that problem (93) is solvable for any $\in L^{2}(\Omega), g \in L^{2}(\partial \Omega)$.

Example 36. Consider the case when $\rho=\alpha_{2} \equiv 0$ in (93); that is, consider the following boundary value problem:

$$
\begin{array}{cc}
-\Delta_{p} u+\alpha_{1}(u)=f & \text { in } \Omega, \\
b(x)|\nabla u|^{p-2} \partial_{n} u=g & \text { on } \Gamma .
\end{array}
$$

Then, by Theorem 31, this problem has a weak solution if

$$
\int_{\Omega} f d x+\int_{\partial \Omega} g \frac{d \sigma}{b} \in \lambda_{1} \operatorname{int}\left(\mathscr{R}\left(\alpha_{1}\right)\right)
$$

which yields the classical Landesman-Lazer result (see (7)) for $g \equiv 0$ and $p=2$.

Example 37. Let us now consider the case when $\alpha_{1} \equiv \alpha$ and $\alpha_{2} \equiv 0$, where $\alpha$ is a continuous, odd, and nondecreasing function on $\mathbb{R}$ such that $\alpha(0)=0$. The problem

$$
\begin{aligned}
-\Delta_{p} u+\alpha(u) & =f, \quad \text { in } \Omega, \\
b(x)|\nabla u|^{p-2} \partial_{n} u-\rho b(x) \Delta_{q, \Gamma} u=g, & \text { on } \partial \Omega,
\end{aligned}
$$

has a weak solution if

$$
\int_{\Omega} f d x+\int_{\partial \Omega} g \frac{d \sigma}{b} \in \lambda_{2} \operatorname{int}(\mathscr{R}(\alpha)) .
$$

Let us now choose $\alpha(s)=\arctan (s)$ in (140). Then, it is easy to check that

$$
\begin{array}{r}
\Lambda(t):=\int_{0}^{|t|} \alpha(s) d s=|t| \arctan (|t|)-\frac{1}{2} \ln \left(1+t^{2}\right), \\
t \in \mathbb{R},
\end{array}
$$

is monotone increasing on $\mathbb{R}_{+}$and that it satisfies $\Lambda(2 t) \leq$ $C_{2} \Lambda(t), \forall t \in \mathbb{R}$, for some constant $C_{2}>1$. Therefore, (141) becomes the necessary and sufficient condition

$$
\left|\frac{1}{\lambda_{2}}\left(\int_{\Omega} f d x+\int_{\partial \Omega} g \frac{d \sigma}{b}\right)\right|<\frac{\pi}{2} .
$$

\section{A Priori Estimates}

Let $\Omega \subset \mathbb{R}^{N}$ be a bounded Lipschitz domain with boundary $\partial \Omega$. Recall that $1<p, q<\infty, \rho \in\{0,1\}$, and $b \in L^{\infty}(\partial \Omega)$ with $b(x) \geq b_{0}>0$, for some constant $b_{0}$. We consider the nonlinear elliptic boundary value problem formally given by

$$
\begin{aligned}
& -\Delta_{p} u+\alpha_{1}(x, u)+|u|^{p-2} u=f, \quad \text { in } \Omega \\
& -\rho b(x) \Delta_{q, \Gamma} u+\rho b(x)|u|^{q-2} u+b(x)|\nabla u|^{p-2} \partial_{n} u \\
& +\alpha_{2}(x, u)=g, \quad \text { on } \partial \Omega,
\end{aligned}
$$

where $f \in L^{p_{1}}(\Omega)$ and $g \in L^{q_{1}}(\partial \Omega)$ for some $1 \leq p_{1}, q_{1} \leq \infty$. If $\rho=0$, then the boundary conditions in (144) are of Robin type. Existence and regularity of weak solutions for this case have been obtained in [25] for $p=2$ (see also [26] for the linear case) and for general $p$ in [27]. Therefore, we will concentrate our attention to the case $\rho=1$ only; in this case, the boundary condition in (144) is a generalized WentzellRobin boundary condition. For the sake of simplicity, from now on we will also take $b \equiv 1$.

5.1. General Assumptions. Throughout this section, we assume that the functions $\alpha_{1}: \Omega \times \mathbb{R} \rightarrow \mathbb{R}$ and $\alpha_{2}: \partial \Omega \times \mathbb{R} \rightarrow$ $\mathbb{R}$ satisfy the following conditions. 
Assumption 38.

$$
\begin{gathered}
\alpha_{j}(x, \cdot) \text { is odd and strictly increasing, } \\
\alpha_{j}(x, 0)=0, \quad \alpha_{j}(x, \cdot) \text { is continuous, } \\
\lim _{t \rightarrow 0} \frac{\alpha_{j}(x, t)}{t}=0, \\
\lim _{t \rightarrow \infty} \frac{\alpha_{j}(x, t)}{t}=\infty,
\end{gathered}
$$

for $\lambda_{N}$, a.e. $x \in \Omega$ if $j=1$, and $\sigma$, a.e. $x \in \partial \Omega$ if $j=2$.

Since $\alpha_{j}(x, \cdot)$ are strictly increasing for $\lambda_{N}$, a.e. $x \in \Omega$ if $j=1$, and $\sigma$, a.e. $x \in \partial \Omega$ if $j=2$, then they have inverses which we denote by $\widetilde{\alpha}_{j}(x, \cdot)$ (cf. also Section 4$)$. We define the functions $\Lambda_{1}, \widetilde{\Lambda}_{1}: \Omega \times \mathbb{R} \rightarrow[0, \infty)$, and $\Lambda_{2}, \widetilde{\Lambda}_{2}: \partial \Omega \times \mathbb{R} \rightarrow$ $[0, \infty)$ by

$$
\begin{aligned}
& \Lambda_{j}(x, t):=\int_{0}^{|t|} \alpha_{j}(x, s) d s, \\
& \widetilde{\Lambda}_{j}(x, t):=\int_{0}^{|t|} \widetilde{\alpha}_{j}(x, s) d s .
\end{aligned}
$$

Then, it is clear that, for $\lambda_{N}$, a.e. $x \in \Omega$ if $j=1$, and $\sigma$, a.e. $x \in$ $\partial \Omega$ if $j=2, \Lambda_{j}(x, \cdot)$ and $\widetilde{\Lambda}_{j}(x, \cdot)$ are differentiable, monotone, and convex with $\Lambda_{j}(x, 0)=\widetilde{\Lambda}_{j}(x, 0)=0$. Furthermore, $\Lambda_{j}(x, \cdot)$ is an $\mathcal{N}$-function and $\widetilde{\Lambda}_{j}(x, \cdot)$ is its complementary $\mathscr{N}$-function. The function $\widetilde{\Lambda}_{j}$ is then the complementary Musielak-Orlick function of $\Lambda_{j}$ in the sense of Young (see Definition 5).

Assumption 39. We assume, for $\lambda_{N}$, a.e. $x \in \Omega$ if $j=1$, and $\sigma$, a.e. $x \in \partial \Omega$ if $j=2$, that $\Lambda_{j}(x, \cdot)$ and $\widetilde{\Lambda}_{j}(x, \cdot)$ satisfy the $\left(\triangle_{2}\right)$-condition in the sense of Definition 7.

It follows from Assumption 39 that there exist two constants $c_{1}, c_{2} \in(0,1]$ such that for $\lambda_{N}$, a.e. $x \in \Omega$ if $j=1$, and $\sigma$, a.e. $x \in \partial \Omega$ if $j=2$, and for all $t \in \mathbb{R}$

$$
c_{j} t \alpha_{j}(x, t) \leq \Lambda_{j}(x, t) \leq t \alpha_{j}(x, t) .
$$

Next, let

$$
\begin{aligned}
& L_{\Lambda_{1}}(\Omega):=\{u: \Omega \\
& \left.\longrightarrow \mathbb{R} \text { measurable: } \int_{\Omega} \Lambda_{1}(x, u) d x<\infty\right\} \\
& L_{\Lambda_{2}}(\partial \Omega):=\{u: \partial \Omega \\
& \left.\quad \longrightarrow \mathbb{R} \text { measurable: } \int_{\partial \Omega} \Lambda_{2}(x, u) d \sigma<\infty\right\} .
\end{aligned}
$$

Since $\Lambda_{j}(x, \cdot)$ and $\widetilde{\Lambda}_{j}(x, \cdot)$ satisfy the $\left(\Delta_{2}\right)$-condition, it follows from [18, Theorem 8.19] that $L_{\Lambda_{1}}(\Omega)$ and $L_{\Lambda_{2}}(\partial \Omega)$, endowed, respectively, with the norms

$$
\begin{aligned}
&\|u\|_{\Lambda_{1}, \Omega}:=\inf \left\{k>0: \int_{\Omega} \Lambda_{1}\left(x, \frac{u(x)}{k}\right) d x \leq 1\right\} \\
&\|u\|_{\Lambda_{2}, \partial \Omega}:=\inf \left\{k>0: \int_{\partial \Omega} \Lambda_{2}\left(x, \frac{u(x)}{k}\right) d \sigma \leq 1\right\}
\end{aligned}
$$

are reflexive Banach spaces. Moreover, by [18, Section 8.11, p. 234], the following generalized versions of Hölder's inequality will also become useful in the sequel

$$
\begin{gathered}
\left|\int_{\Omega} u v d x\right| \leq 2\|u\|_{\Lambda_{1}, \Omega}\|v\|_{\widetilde{\Lambda}_{1}, \Omega}, \\
\left|\int_{\partial \Omega} u v d \sigma\right| \leq 2\|u\|_{\Lambda_{2}, \partial \Omega}\|v\|_{\widetilde{\Lambda}_{2}, \partial \Omega} .
\end{gathered}
$$

5.2. Existence and Uniqueness of Weak Solutions of Perturbed Equations. Let

$$
\begin{aligned}
\mathscr{V} & :=\left\{U:=\left(u,\left.u\right|_{\partial \Omega}\right): u \in W^{1, p}(\Omega) \cap L_{\Lambda_{1}}(\Omega), u_{\mid \partial \Omega}\right. \\
& \left.\in W^{1, q}(\partial \Omega) \cap L_{\Lambda_{2}}(\partial \Omega)\right\}
\end{aligned}
$$

Then, for every $1<p, q<\infty, \mathscr{V}$ endowed with the norm

$$
\begin{aligned}
\|U\|_{\mathscr{V}}= & \|u\|_{W^{1, p}(\Omega)}+\|u\|_{\Lambda_{1}, \Omega}+\|u\|_{W^{1, q}(\partial \Omega)} \\
& +\|u\|_{\Lambda_{2}, \partial \Omega}
\end{aligned}
$$

is a reflexive Banach space. Recall that $\rho=1$. Throughout the following, we denote by $\mathscr{V}^{\prime}$ the dual of $\mathscr{V}$.

Definition 40. A function $U=\left(u,\left.u\right|_{\partial \Omega}\right) \in \mathscr{V}$ is said to be a weak solution of (144), if for every $V \in \mathscr{V}=\left(v,\left.v\right|_{\partial \Omega}\right)$

$$
\mathscr{A}(U, V)=\int_{\Omega} f v d x+\int_{\partial \Omega} g v d \sigma,
$$

provided that the integrals on the right-hand side exist. Here

$$
\begin{aligned}
\mathscr{A}(U, V):= & \int_{\Omega}|\nabla u|^{p-2} \nabla u \cdot \nabla v d x+\int_{\Omega}|u|^{p-2} u v d x \\
& +\int_{\Omega} \alpha_{1}(x, u) v d x+\int_{\partial \Omega}\left|\nabla_{\Gamma} u\right|^{q-2} \nabla_{\Gamma} u \\
& \cdot \nabla_{\Gamma} v d \sigma+\int_{\partial \Omega}|u|^{q-2} u v d \sigma \\
& +\int_{\partial \Omega} \alpha_{2}(x, u) v d \sigma .
\end{aligned}
$$

Lemma 41. Assume Assumptions 38 and 39. Let $1<p, q<\infty$ and $U \in \mathscr{V}$ be fixed. Then the functional $V \mapsto \mathscr{A}(U, V)$ belongs to $\mathscr{V}^{\prime}$. Moreover, $\mathscr{A}$ is strictly monotone, hemicontinuous, and coercive. 
Proof. Let $U=\left(u,\left.u\right|_{\partial \Omega}\right) \in \mathscr{V}$ be fixed. It is clear that $\mathscr{A}(U, \cdot)$ is linear. Let $V=\left(v,\left.v\right|_{\partial \Omega}\right) \in \mathscr{V}$. Then, exploiting (150), we obtain

$$
\begin{aligned}
& |\mathscr{A}(U, V)| \\
& \leq\|u\|_{W^{1, p}(\Omega)}^{p-1}\|v\|_{W^{1, p}(\Omega)}+\|u\|_{W^{1, q}(\partial \Omega)}^{q-1}\|v\|_{W^{1, q}(\partial \Omega)} \\
& \quad+2 \max \left\{1, \int_{\Omega} \widetilde{\Lambda}_{1}\left(x, \alpha_{1}(x, u)\right) d x\right\}\|v\|_{\Lambda_{1}, \Omega} \\
& \quad+2 \max \left\{1, \int_{\partial \Omega} \widetilde{\Lambda}_{2}\left(x, \alpha_{2}(x, u)\right) d \sigma\right\}\|v\|_{\Lambda_{2}, \partial \Omega} \\
& \leq K(U)\|V\|_{\mathscr{V}},
\end{aligned}
$$

where

$$
\begin{aligned}
K(U):= & \|u\|_{W^{1, p}(\Omega)}^{p-1} \\
& +2 \max \left\{1, \int_{\Omega} \widetilde{\Lambda}_{1}\left(x, \alpha_{1}(x, u)\right) d x\right\} \\
& +\|u\|_{W^{1, q}(\partial \Omega)}^{q-1} \\
& +2 \max \left\{1, \int_{\partial \Omega} \widetilde{\Lambda}_{2}\left(x, \alpha_{2}(x, u)\right) d \sigma\right\} .
\end{aligned}
$$

This shows $\mathscr{A}(U, \cdot) \in \mathscr{V}^{\prime}$, for every $U \in \mathscr{V}$.

Next, let $U, V \in \mathscr{V}$. Then, using (59) and the fact that $\alpha_{j}(x, \cdot)$ are monotone nondecreasing, that is, $\left(\alpha_{j}(x, t)-\right.$ $\left.\alpha_{j}(x, s)\right)(t-s) \geq 0$, for all $t, s \in \mathbb{R}$, we obtain

$$
\begin{aligned}
\mathscr{A} & (U, U-V)-\mathscr{A}(V, U-V) \\
& =\int_{\Omega}\left(|\nabla u|^{p-2} \nabla u-|\nabla v|^{p-2} \nabla v\right) \cdot \nabla(u-v) d x \\
& +\int_{\Omega}\left(|u|^{p-2} u-|v|^{p-2} v\right)(u-v) d x \\
& +\int_{\Omega}\left(\alpha_{1}(x, u)-\alpha_{1}(x, v)\right)(u-v) d x \\
& +\int_{\partial \Omega}\left(|u|^{q-2} u-|v|^{q-2} v\right)(u-v) d \sigma \\
& +\int_{\partial \Omega}\left(\left|\nabla_{\Gamma} u\right|^{q-2} \nabla_{\Gamma} u-\left|\nabla_{\Gamma} v\right|^{q-2} \nabla v\right) \cdot \nabla_{\Gamma}(u-v) d \sigma \\
& +\int_{\partial \Omega}\left(\alpha 2(x, u)-\alpha_{1}(x, v)\right)(u-v) d \sigma \\
& \geq \int_{\Omega}(|\nabla u|+|\nabla v|)^{p-2}|\nabla(u-v)|^{2} d x \\
& +\int_{\Omega}(|u|+|v|)^{p-2}|u-v|^{2} d x \\
& +\int_{\partial \Omega}\left(\left|\nabla_{\Gamma} u\right|+\left|\nabla_{\Gamma} v\right|\right)^{p-2}\left|\nabla_{\Gamma}(u-v)\right|^{2} d \sigma \\
+ & \int_{\partial \Omega}(|u|+|v|)^{p-2}|u-v|^{2} d \sigma \geq 0 . \\
&
\end{aligned}
$$

This shows that $\mathscr{A}$ is monotone. Estimate (157) also shows that

$$
\mathscr{A}(U, U-V)-\mathscr{A}(V, U-V)>0,
$$

for all $U, V \in V$ with $U \neq V$, that is, $u \neq v$ or $\left.u\right|_{\partial \Omega} \neq\left. v\right|_{\partial \Omega}$. Thus, $\mathscr{A}$ is strictly monotone.

The continuity of the norm function and the continuity of $\alpha_{j}(x, \cdot), j=1,2$ imply that $\mathscr{A}$ is hemicontinuous.

Finally, since $\Lambda_{j}$ and $\widetilde{\Lambda}_{j}$ satisfy the $\left(\triangle_{2}^{0}\right)$-condition, from Proposition 12 and Corollary 13, it follows that

$$
\begin{array}{r}
\lim _{\|u\|_{\Lambda_{1}, \Omega} \rightarrow+\infty} \frac{\int_{\Omega} u \alpha_{1}(x, u) d x}{\|u\|_{\Lambda_{1}, \Omega}}=+\infty, \\
\lim _{\|u\|_{\Lambda_{2}, \partial \Omega} \rightarrow+\infty} \frac{\int_{\partial \Omega} u \alpha_{2}(x, u) d \sigma}{\|u\|_{\Lambda_{2}, \partial \Omega}}=+\infty .
\end{array}
$$

Consequently, we deduce

$$
\lim _{\|U\|_{\mathscr{V}} \rightarrow+\infty} \frac{\mathscr{A}(U, U)}{\|U\|_{\mathscr{V}}}=+\infty
$$

which shows that $\mathscr{A}$ is coercive. The proof of the lemma is finished.

The following result is concerned with the existence and uniqueness of weak solutions to problem (144).

Theorem 42. Assume Assumptions 38 and 39. Let $1<p, q<$ $\infty, p_{1} \geq p *$, and $q_{1} \geq q *$, where $p *:=p /(p-1)$ and $q *:=$ $q /(q-1)$. Then, for every $(f, g) \in X^{p_{1}, q_{1}}(\bar{\Omega}, \mu)$, there exists a unique function $U \in \mathscr{V}$ which is a weak solution to (144).

Proof. Let $\langle\cdot, \cdot\rangle$ denote the duality between $\mathscr{V}$ and $\mathscr{V}^{\prime}$. Then, from Lemma 41, it follows that, for each $U \in \mathscr{V}$, there exists $A(U) \in \mathscr{V}^{\prime}$ such that

$$
\mathscr{A}(U, V)=\langle A(U), V\rangle,
$$

for every $V \in \mathscr{V}$. Hence, this relation defines an operator $A: \mathscr{V} \rightarrow \mathscr{V}^{\prime}$, which is bounded by (155). Exploiting Lemma 41 once again, it is easy to see that $A$ is monotone and coercive. It follows from Browder's theorem (see, e.g., [28, Theorem 5.3.22]) that $A(\mathscr{V})=\mathscr{V}^{\prime}$. Therefore, for every $F \in \mathscr{V}^{\prime}$ there exists $U \in \mathscr{V}$ such that $A(U)=F$; that is, for every $V \in \mathscr{V}$

$$
\langle A(U), V\rangle=\mathscr{A}(U, V)=\langle V, F\rangle .
$$

Since $W^{1, p}(\Omega) \hookrightarrow L^{p}(\Omega)$ and $W^{1, q}(\partial \Omega) \hookrightarrow L^{q}(\partial \Omega)$ with dense injection, by duality, we have $X^{p *, q *}(\bar{\Omega}, \mu) \hookrightarrow \mathscr{V}^{\prime}$. Since $\Omega$ is bounded and $\sigma(\partial \Omega)<\infty$, we obtain that

$$
X^{p_{1}, q_{1}}(\bar{\Omega}, \mu) \hookrightarrow X^{p *, q *}(\bar{\Omega}, \mu) \hookrightarrow \mathscr{V}^{\prime} .
$$

This shows the existence of weak solutions. The uniqueness follows from the fact that $\mathscr{A}$ is strictly monotone (cf. Lemma 41). This completes the proof of the theorem. 
Corollary 43. Let the assumptions of Theorem 42 be satisfied. Let

$$
\begin{aligned}
p_{h} & :=\frac{N p}{N(p-1)+p}, \\
q_{h} & :=\frac{p(N-1)}{N(p-1)}, \\
q_{k} & :=\frac{q(N-1)}{N(q-1)+1} .
\end{aligned}
$$

(a) Let $1<p<N, 1<q<p(N-1) / N$, $p_{1} \geq p_{h}$, and $q_{1} \geq$ $q_{k}$. Then, for every $(f, g) \in X^{p_{1}, q_{1}}(\Omega, \mu)$, there exists a function $U \in \mathscr{V}$ which is the unique weak solution to (144).

(b) Let $1<q<N-1,1<p<N q /(N-1), p_{1} \geq$ $p_{h}$, and $q_{1} \geq q_{h}$. Then, for every $(f, g) \in X^{p_{1}, q_{1}}(\Omega, \mu)$, there exists a function $U \in \mathscr{V}$ which is the unique weak solution to (144).

Proof. We first prove (a). Let $1<p<N$ and $1<q<p$ (N1)/N and let $p_{1} \geq p_{h}$ and $q_{1} \geq q_{k}$, where $p_{h}$ and $q_{k}$ are given by $(164)$. Let $p_{s}:=N p /(N-p)$ and $q_{t}:=(N-1) q /(N-1-q)$. Since $W^{1, p}(\Omega) \hookrightarrow L^{p_{s}}(\Omega)$ and $W^{1, q}(\partial \Omega) \hookrightarrow L^{q_{t}}(\partial \Omega)$ with dense injection, then, by duality, $X^{p_{h}, q_{k}}(\bar{\Omega}, \mu) \hookrightarrow \mathscr{V}^{\prime}$, where $1 / p_{s}+1 / p_{h}=1$ and $1 / q_{t}+1 / q_{k}=1$. Since $\mu(\bar{\Omega})<\infty$, we have that

$$
X^{p_{1}, q_{1}}(\bar{\Omega}, \mu) \hookrightarrow X^{p_{h}, q_{h}}(\bar{\Omega}, \mu) \hookrightarrow \mathscr{V}^{\prime} .
$$

Hence, for every $F:=(f, g) \in X^{p_{1}, q_{1}}(\bar{\Omega}, \mu) \hookrightarrow \mathscr{V}^{\prime}$, there exists $U \in \mathscr{V}$ such that for every $V \in \mathscr{V}$

$$
\langle A(U), V\rangle=\mathscr{A}(U, V)=\int_{\Omega} f v d x+\int_{\partial \Omega} g v d \sigma .
$$

The uniqueness of the weak solution follows again from the fact that $\mathscr{A}$ is strictly monotone.

In order to prove the second part, we use the embeddings $W^{1, p}(\Omega) \hookrightarrow L^{p_{s}}(\Omega), W^{1, p}(\Omega) \hookrightarrow L^{q_{s}}(\partial \Omega)$ and proceed exactly as above. We omit the details.

5.3. Properties of the Solution Operator of the Perturbed Equation. In the sequel, we establish some interesting properties of the solution operator $A$ to problem (144). We begin by assuming the following.

Assumption 44. Suppose that $\alpha_{j}, j=1,2$, satisfy the following conditions:

there are constants $c_{j} \in(0,1]$ such that

$$
c_{j}\left|\alpha_{j}(x, \xi-\eta)\right| \leq\left|\alpha_{j}(x, \xi)-\alpha_{j}(x, \eta)\right|
$$

\section{$\forall \xi, \eta \in \mathbb{R}$}

Theorem 45. Assume Assumptions 38, 39, and 44. Let $p, q \geq 2$ and let $A: \mathscr{V} \rightarrow \mathscr{V}^{\prime}$ be the continuous and bounded operator constructed in the proof of Theorem 42. Then $A$ is injective and, hence, invertible and its inverse $A^{-1}$ is also continuous and bounded.
Proof. First, we remark that since

$$
\left(\alpha_{j}(x, t)-\alpha_{j}(x, s)\right)(t-s) \geq 0, \quad \forall t, s \in \mathbb{R},
$$

for $\lambda_{N}$, a.e. $x \in \Omega$ if $j=1$, and $\sigma$, a.e. $x \in \partial \Omega$ if $j=2$, it follows from (167) that, for all $t, s \in \mathbb{R}$,

$$
\left(\alpha_{j}(x, t)-\alpha_{j}(x, s)\right)(t-s) \geq c_{j} \alpha_{j}(x, t-s) \cdot(t-s) .
$$

Let $U, V \in \mathscr{V}$ and $p, q \in[2, \infty)$. Then, exploiting (60), (169), and the $\left(\triangle_{2}\right)$-condition, we obtain

$$
\begin{aligned}
& \langle A(U)-A(V), U-V\rangle=\mathscr{A}(U, U-V)-\mathscr{A}(V, U \\
& -V)=\int_{\Omega}\left(|\nabla u|^{p-2} \nabla u-|\nabla v|^{p-2} \nabla v\right) \\
& +\nabla(u-v) d x \\
& +\int_{\Omega}\left(|u|^{p-2} u-|v|^{p-2} v\right)(u-v) d x \\
& +\int_{\Omega}\left(\alpha_{1}(x, u)-\alpha_{1}(x, v)\right)(u-v) d x \\
& +\int_{\partial \Omega}\left(\left|\nabla_{\Gamma} u\right|^{q-2} \nabla_{\Gamma} u-\left|\nabla_{\Gamma} v\right|^{q-2} \nabla_{\Gamma} v\right) \\
& +\nabla_{\Gamma}(u-v) d \sigma \\
& +\int_{\partial \Omega}\left(|u|^{q-2} u-|v|^{q-2} v\right)(u-v) d \sigma \\
& +\int_{\partial \Omega}\left(\alpha_{2}(x, u)-\alpha_{2}(x, v)\right)(u-v) d \sigma \geq \| u \\
& +v\left\|_{W^{1, p}(\Omega)}^{p}+c_{1} \int_{\Omega} \Lambda_{1}(x, u-v) d x+\right\| u \\
& -v \|_{W^{1, q}(\partial \Omega)}^{q}+c_{2} \int_{\partial \Omega} \Lambda_{2}(x, u-v) d \sigma .
\end{aligned}
$$

This implies that $\langle A(U)-A(V), U-V\rangle>0$, for all $U, V \in \mathscr{V}$ with $U \neq V$ (that is, $u \neq v$, or $\left.u\right|_{\partial \Omega} \neq\left. v\right|_{\partial \Omega}$ ). Therefore, the operator $A$ is injective and, hence, $A^{-1}$ exists. Since for every $U \in \mathscr{V}$

$$
\mathscr{A}(U, U)=\langle A(U), U\rangle \leq\|A(U)\|_{\mathscr{V}^{\prime}}\|U\|_{\mathscr{V}},
$$

from the coercivity of $\mathscr{A}$ (see (160)), it is not difficult to see that

$$
\lim _{\|U\|_{\mathscr{V} \rightarrow+\infty}}\|A(U)\|_{\mathscr{V}^{\prime}}=+\infty
$$

Thus, $A^{-1}: \mathscr{V}^{\prime} \rightarrow \mathscr{V}$ is bounded.

Next, we show that $A^{-1}: \mathscr{V}^{\prime} \rightarrow \mathscr{V}$ is continuous. Assume that $A^{-1}$ is not continuous. Then there is a sequence $F_{n} \in \mathscr{V}^{\prime}$ with $F_{n} \rightarrow F$ in $\mathscr{V}^{\prime}$ and a constant $\delta>0$ such that

$$
\left\|A^{-1}\left(F_{n}\right)-A^{-1}(F)\right\|_{\mathscr{V}} \geq \delta
$$


for all $n \in \mathbb{N}$. Let $U_{n}:=A^{-1}\left(F_{n}\right)$ and $U=A^{-1}(F)$. Since $\left\{F_{n}\right\}$ is a bounded sequence and $A^{-1}$ is bounded, we have that $\left\{U_{n}\right\}$ is bounded in $\mathscr{V}$. Thus, we can select a subsequence, which we still denote by $\left\{U_{n}\right\}$, which converges weakly to some function $V \in \mathscr{V}$. Since $A\left(U_{n}\right)-A(V) \rightarrow F-A(V)$ strongly in $\mathscr{V}$ and $U_{n}-V$ converges weakly to zero in $\mathscr{V}$, we deduce

$$
\lim _{n \rightarrow \infty}\left\langle A\left(U_{n}\right)-A(V), U_{n}-V\right\rangle=0 .
$$

From (170) and (174), it follows that

$$
\begin{array}{r}
\lim _{n \rightarrow \infty}\left\|u_{n}-v\right\|_{W^{1, p}(\Omega)}=0, \\
\lim _{n \rightarrow \infty} \int_{\Omega} \Lambda_{1}\left(x, u_{n}-v\right) d x=0,
\end{array}
$$

while

$$
\begin{array}{r}
\lim _{n \rightarrow \infty}\left\|u_{n}-v\right\|_{W^{1, q}(\partial \Omega)}=0, \\
\lim _{n \rightarrow \infty} \int_{\partial \Omega} \Lambda_{2}\left(x, u_{n}-v\right) d \sigma=0 .
\end{array}
$$

Therefore, $U_{n} \rightarrow V$ strongly in $\mathscr{V}$. Since $A$ is continuous and

$$
\begin{gathered}
F_{n}=A\left(U_{n}\right) \longrightarrow \\
A(V)=F=A(U),
\end{gathered}
$$

it follows from the injectivity of $A$ that $U=V$. This shows that

$$
\lim _{n \rightarrow \infty}\left\|A^{-1}\left(F_{n}\right)-A^{-1}(F)\right\|_{\mathscr{V}}=\lim _{n \rightarrow \infty}\left\|U_{n}-U\right\|_{\mathscr{V}}=0,
$$

which contradicts (173). Hence, $A^{-1}: \mathscr{V}^{\prime} \rightarrow \mathscr{V}$ is continuous. The proof is finished.

Corollary 46. Let the assumptions of Theorem 45 be satisfied. Let $p_{h}, q_{h}$, and $q_{k}$ be as in (164) and let $A: \mathscr{V} \rightarrow \mathscr{V}^{\prime}$ be the continuous and bounded operator constructed in the proof of Theorem 42.

(a) If $2 \leq p<N, 2 \leq q<p(N-1) / N, p_{1} \geq$ $p_{h}$, and $q_{1} \geq q_{k}$, then $A^{-1}: X^{p_{1}, q_{1}}(\bar{\Omega}, \mu) \rightarrow$ $X^{p_{s}}, q_{t}(\bar{\Omega}, \mu)$ is continuous and bounded. Moreover, $A^{-1}: X^{p_{1}, q_{1}}(\bar{\Omega}, \mu) \rightarrow \mathscr{V} \cap X^{r, s}(\bar{\Omega}, \mu)$ is compact for every $r \in\left(1, p_{s}\right)$ and $s \in\left(1, q_{s}\right)$.

(b) If $2 \leq q<N-1,2 \leq p<q N /(N-1), p_{1} \geq p_{h}$, and $q_{1} \geq q_{h}$, then the operator $A^{-1}: X^{p_{1}, q_{1}}(\bar{\Omega}, \mu) \rightarrow$ $X^{p_{s}, q_{s}}(\bar{\Omega}, \mu)$ is continuous and bounded. Moreover, $A^{-1}: X^{p_{1}, q_{1}}(\bar{\Omega}, \mu) \rightarrow \mathscr{V} \cap X^{r, s}(\bar{\Omega}, \mu)$ is compact for every $r \in\left(1, p_{s}\right)$ and $s \in\left(1, q_{s}\right)$.

Proof. We only prove the first part. The second part of the proof follows by analogy and is left to the reader. Let $2 \leq$ $p<N, 2 \leq q<p(N-1) / N, p_{1} \geq p_{h}$, and $q_{1} \geq q_{k}$ and let $F \in X^{p_{1}, q_{1}}(\bar{\Omega}, \mu)$. Proceeding exactly as in the proof of Theorem 45 , we obtain

$$
\begin{aligned}
\left\|A^{-1}(F)\right\|_{p_{s}, q_{t}} & \leq C_{1}\left\|A^{-1}(F)\right\|_{\mathscr{V}} \leq C\|F\|_{\mathscr{V}^{\prime}} \\
& \leq C_{2}\|F\|_{p_{1}, q_{1}} .
\end{aligned}
$$

Hence, the operator $A^{-1}: X^{p_{1}, q_{1}}(\bar{\Omega}, \mu) \rightarrow X^{p_{s}, q_{t}}(\bar{\Omega}, \mu)$ is bounded. Finally, using the facts that $X^{p_{1}, q_{1}}(\bar{\Omega}, \mu) \hookrightarrow \mathscr{V}^{\prime}$, $A^{-1}: \mathscr{V}^{\prime} \rightarrow \mathscr{V}$ is continuous and $\mathscr{V} \hookrightarrow X^{p_{s}, q_{t}}(\bar{\Omega}, \mu)$, we easily deduce that $A^{-1}: X^{p_{1}, q_{1}}(\bar{\Omega}, \mu) \rightarrow X^{p_{s}, q_{t}}(\bar{\Omega}, \mu)$ is continuous.

Now, let $1<r<p_{s}$ and $1<s<q_{s}$. Since the injection $\mathscr{V} \hookrightarrow X^{r, s}(\bar{\Omega}, \mu)$ is compact, then, by duality, the injection $X^{r^{\prime}, s^{\prime}}(\bar{\Omega}, \mu) \hookrightarrow \mathscr{V}^{\prime}$ is compact for every $r^{\prime}>p_{s}^{\prime}=p_{h}$ and $s^{\prime}>q_{s}^{\prime}=q_{h}$. This, together with the fact that $A^{-1}: \mathscr{V}^{\prime} \rightarrow \mathscr{V}$ is continuous and bounded, implies that $A^{-1}: X^{p_{1}, q_{1}}(\bar{\Omega}, \mu) \rightarrow$ $\mathscr{V}$ is compact for every $p_{1}>p_{h}$ and $q_{1}>q_{h}$.

It remains to show that $A^{-1}$ is also compact as a map into $X^{r, s}(\bar{\Omega}, \mu)$ for every $r \in\left(1, p_{s}\right)$ and $s \in\left(1, q_{s}\right)$. Since $A^{-1}$ is bounded, we have to show that the image of every bounded set $\mathscr{B} \subset \mathbb{X}^{p_{1}, q_{1}}(\Omega, \mu)$ is relatively compact in $X^{r, s}(\bar{\Omega}, \mu)$ for every $r \in\left(1, p_{s}\right)$ and $s \in\left(1, q_{s}\right)$. Let $U_{n}$ be a sequence in $A^{-1}(\mathscr{B})$. Let $F_{n}=A\left(U_{n}\right) \in \mathscr{B}$. Since $\mathscr{B}$ is bounded, then the sequence $F_{n}$ is bounded. Since $A^{-1}$ is compact as a map into $\mathscr{V}$, it follows that there is a subsequence $F_{n_{k}}$ such that $A^{-1}\left(F_{n_{k}}\right) \rightarrow U \in \mathscr{V}$. We may assume that $U_{n}=A^{-1}\left(F_{n}\right) \rightarrow$ $U$ in $\mathscr{V}$ and, hence, in $X^{p, p}(\bar{\Omega}, \mu)$. It remains to show that $U_{n} \rightarrow U$ in $X^{r, s}(\bar{\Omega}, \mu)$. Let $r \in\left[p, p_{s}\right)$ and $s \in\left[p, q_{s}\right)$. Since $U_{n}:=\left(u_{n},\left.u_{n}\right|_{\partial \Omega}\right)$ is bounded in $X^{p_{s}, q_{s}}(\bar{\Omega}, \mu)$, a standard interpolation inequality shows that there exists $\tau \in(0,1)$ such that

$$
\begin{aligned}
\left\|\left|U_{n}-U_{m} \|\right|_{r, s}\right. & \leq\left.\left\|\left|U_{n}-U_{m}\left\|\left.\right|_{p, p} ^{\tau}\right\|\right| U_{n}-U_{m}\right\|\right|_{p_{s}, q_{s}} ^{1-\tau} \\
& \leq C\left\|\left|U_{n}-U_{m} \|\right|_{p, p}^{\tau} .\right.
\end{aligned}
$$

As $U_{n}$ converges in $X^{p, p}(\bar{\Omega}, \mu)$, it follows from the preceding inequality that $U_{n}$ is a Cauchy sequence in $X^{r, s}(\bar{\Omega}, \mu)$ and therefore converges in $X^{r, s}(\bar{\Omega}, \mu)$. Hence, $A^{-1}: X^{p_{1}, q_{1}}(\bar{\Omega}$, $\mu) \rightarrow \mathscr{V} \cap X^{r, s}(\bar{\Omega}, \mu)$ is compact for every $r \in\left[p, p_{s}\right)$ and $s \in\left[p, q_{s}\right)$. The case $r, s \in(1, p)$ follows from the fact that $X^{p, p}(\bar{\Omega}, \mu) \hookrightarrow X^{r, s}(\bar{\Omega}, \mu)$ and the proof is finished

5.4. Statement and Proof of the Main Result. We will now establish under what conditions the operator $A^{-1}$ maps $X^{p_{1}, q_{1}}(\bar{\Omega}, \mu)$ boundedly and continuously into $X^{\infty}(\bar{\Omega}, \mu)$. The following is the main result of this section.

Theorem 47. Let the assumptions of Theorem 45 be satisfied.

(a) Suppose $2 \leq p<N$ and $2 \leq q<\infty$. Let

$$
\begin{aligned}
& p_{1}>\frac{p_{s}}{p_{s}-p}=\frac{N}{p}, \\
& q_{1}>\frac{q_{s}}{q_{s}-p}=\frac{N-1}{p-1} .
\end{aligned}
$$

Let $f \in L^{p_{1}}(\Omega), g \in L^{q_{1}}(\partial \Omega)$ and $U, V \in \mathscr{V}$ be such that for every function $\Phi=\left(\varphi,\left.\varphi\right|_{\partial \Omega}\right) \in \mathscr{V}$

$$
\mathscr{A}(U, \Phi)-\mathscr{A}(V, \Phi)=\int_{\Omega} f \varphi d x+\int_{\partial \Omega} g \varphi d \sigma
$$


Then there is a constant $C=C(N, p, q, \Omega)>0$ such that

$$
\left\||U-V \||_{\infty}^{p-1} \leq C\left(\|f\|_{p_{1}, \Omega}+\|g\|_{q_{1}, \partial \Omega}\right) .\right.
$$

(b) Suppose $2 \leq p=q<N-1$. Let

$$
\begin{aligned}
& p_{1}>\frac{p_{s}}{p_{s}-p}=\frac{N}{p}, \\
& q_{1}>\frac{p_{t}}{p_{t}-p}=\frac{N-1}{p} .
\end{aligned}
$$

Let $f \in L^{p_{1}}(\Omega), g \in L^{q_{1}}(\partial \Omega)$ and $U, V \in \mathscr{V}$ satisfy (182). Then there is a constant $C=C(N, p, q, \Omega)>0$ such that

$$
\left.\|U-V\|\right|_{\infty} ^{p-1} \leq C\left(\|f\|_{p_{1}, \Omega}+\|g\|_{q_{1}, \partial \Omega}\right) .
$$

Proof. Let $U, V \in \mathscr{V}$ satisfy (182). Let $k \geq 0$ be a real number and set

$$
\begin{aligned}
w_{k} & :=(|u-v|-k)^{+} \operatorname{sgn}(u-v) \\
W_{k} & :=\left(w_{k},\left.w_{k}\right|_{\partial \Omega}\right), \\
w & :=|u-v| .
\end{aligned}
$$

Let $A_{k}:=\{x \in \bar{\Omega}:|w(x)| \geq k\}$ and $A_{k}^{+}:=\{x \in \bar{\Omega}: w(x) \geq k\}$ and $A_{k}^{-}:=\{x \in \bar{\Omega}: w(x) \leq-k\}$. Clearly $W_{k} \in \mathscr{V}$ and $A_{k}=$ $A_{k}^{+} \cup A_{k}^{-}$. We claim that there exists a constant $C>0$ such that

$$
C \mathscr{A}\left(W_{k}, W_{k}\right) \leq \mathscr{A}\left(U, W_{k}\right)-\mathscr{A}\left(V, W_{k}\right),
$$

for all $U, V \in \mathscr{V}$. Using the definition of the form $\mathscr{A}$, we have

$$
\begin{aligned}
\mathscr{A}(U, & \left.W_{k}\right)-\mathscr{A}\left(V, W_{k}\right) \\
= & \int_{\Omega}\left(|\nabla u|^{p-2} \nabla u-|\nabla v|^{p-2} \nabla v\right) \cdot \nabla w_{k} d x \\
& +\int_{\Omega}\left(|u|^{p-2} u-|v|^{p-2} v\right) w_{k} d x \\
& +\int_{\Omega}\left(\alpha_{1}(x, u)-\alpha_{2}(x, v)\right) w_{k} d x \\
& +\int_{\partial \Omega}\left(|u|^{q-2} u-|v|^{q-2} v\right) w_{k} d \sigma \\
& +\int_{\partial \Omega}\left(\left|\nabla_{\Gamma} u\right|^{p-2} \nabla_{\Gamma} u-\left|\nabla_{\Gamma} v\right|^{p-2} \nabla_{\Gamma} v\right) \cdot \nabla_{\Gamma} w_{k} d \sigma \\
& +\int_{\partial \Omega}\left(\alpha_{2}(x, u)-\alpha_{2}(x, v)\right) w_{k} d \sigma .
\end{aligned}
$$

Since

$$
\nabla w_{k}= \begin{cases}\nabla(u-v) & \text { in } A(k), \\ 0 & \text { otherwise }\end{cases}
$$

we can rewrite (188) as follows:

$$
\begin{aligned}
& \mathscr{A}\left(U, W_{k}\right)-\mathscr{A}\left(V, W_{k}\right) \\
& =\int_{A(k) \cap \Omega}\left(|\nabla u|^{p-2} \nabla u-|\nabla v|^{p-2} \nabla v\right) \\
& \cdot \nabla(u-v) d x \\
& +\int_{A(k) \cap \partial \Omega}\left(\left|\nabla_{\Gamma} u\right|^{q-2} \nabla_{\Gamma} u-\left|\nabla_{\Gamma} v\right|^{q-2} \nabla_{\Gamma} v\right) \\
& +\nabla_{\Gamma}(u-v) d \sigma \\
& +\lambda \int_{A(k) \cap \Omega}\left(|u|^{p-2} u-|v|^{p-2} v\right) w_{k} d x \\
& +\int_{A(k) \cap \Omega}\left(\alpha_{1}(x, u)-\alpha_{1}(x, v)\right) w_{k} d x \\
& +\int_{A(k) \cap \partial \Omega}\left(\alpha_{2}(x, u)-\alpha_{2}(x, v)\right) w_{k} d \sigma .
\end{aligned}
$$

Exploiting inequality (60), from (190) and (169), we deduce that

$$
\begin{aligned}
& \mathscr{A}\left(U, W_{k}\right)-\mathscr{A}\left(V, W_{k}\right) \\
& \geq \int_{A(k) \cap \Omega}\left(\left|\nabla w_{k}\right|^{p}+\left|w_{k}\right|^{p}\right) d x \\
& +\int_{A(k) \cap \Omega} c_{1} \alpha_{1}\left(x, w_{k}\right) w_{k} d x \\
& +\int_{A(k) \cap \Omega}\left(|u|^{p-2} u w_{k}-|v|^{p-2} v w_{k}-\left|w_{k}\right|^{p}\right) d x \\
& +\int_{A(k) \cap \Omega}\left(\alpha_{1}(x, u)-\alpha_{1}(x, v)-c_{1} \alpha_{1}\left(x, w_{k}\right)\right) \\
& +w_{k} d x+\int_{A(k) \cap \partial \Omega}\left(\left|\nabla_{\Gamma} w_{k}\right|^{q}+\left|w_{k}\right|^{q}\right) d \sigma \\
& +\int_{A(k) \cap \partial \Omega} c_{2} \alpha_{2}\left(x, w_{k}\right) w_{k} d \sigma \\
& +\int_{A(k) \cap \partial \Omega}\left(|u|^{q-2} u w_{k}-|v|^{q-2} v w_{k}-\left|w_{k}\right|^{q}\right) d \sigma \\
& +\int_{A(k) \cap \partial \Omega}\left(\alpha_{2}(x, u)-\alpha_{2}(x, v)-c_{2} \alpha_{2}\left(x, w_{k}\right)\right) \\
& +w_{k} d \sigma \geq C \mathscr{A}\left(W_{k}, W_{k}\right) \\
& +\int_{A(k) \cap \Omega}\left(|u|^{p-2} u w_{k}-|v|^{p-2} v w_{k}-\left|w_{k}\right|^{p}\right) d x \\
& +\int_{A(k) \cap \Omega}\left(\alpha_{1}(x, u)-\alpha_{1}(x, v)-c_{1} \alpha_{1}\left(x, w_{k}\right)\right) \\
& +w_{k} d x
\end{aligned}
$$




$$
\begin{aligned}
& +\int_{A(k) \cap \partial \Omega}\left(|u|^{q-2} u w_{k}-|v|^{q-2} v w_{k}-\left|w_{k}\right|^{q}\right) d \sigma \\
& +\int_{A(k) \cap \partial \Omega}\left(\alpha_{2}(x, u)-\alpha_{2}(x, v)-c_{2} \alpha_{2}\left(x, w_{k}\right)\right) \\
& \cdot w_{k} d \sigma
\end{aligned}
$$

where $c_{1}, c_{2}$ are the constants from (169). Using (167) and the fact that $\alpha_{j}(x, \cdot)$ are strictly increasing, for $x \in A_{k}^{+}$, we have

$$
\begin{aligned}
c_{j} \alpha_{j}\left(x, w_{k}(x)\right) & =c_{j} \alpha_{j}(x, u(x)-v(x)-k) \\
& \leq c_{j} \alpha_{j}(x, u(x)-v(x)) \\
& \leq \alpha_{j}(x, u(x))-\alpha_{j}(x, v(x)) .
\end{aligned}
$$

Multiplying this inequality by $w_{k}(x) \geq 0, x \in A_{k}^{+}$, yields

$$
\begin{aligned}
& \left(\alpha_{j}(x, u(x))-\alpha_{j}(x, v(x))-c_{j} \alpha_{j}\left(x, w_{k}(x)\right)\right) \\
& \cdot w_{k}(x) \geq 0 .
\end{aligned}
$$

Similarly, for $x \in A_{k}^{-}$

$$
\begin{aligned}
c_{j} \alpha_{j}\left(x, w_{k}(x)\right) & =c_{j} \alpha_{j}(x, u(x)-v(x)+k) \\
& \geq c_{j} \alpha_{j}(x, u(x)-v(x)) \\
& \geq \alpha_{j}(x, u(x))-\alpha_{j}(x, v(x)) .
\end{aligned}
$$

Hence, multiplying this inequality by $w_{k}(x) \leq 0$, we get

$$
\begin{aligned}
& \left(\alpha_{j}(x, u(x))-\alpha_{j}(x, v(x))-c_{j} \alpha_{j}\left(x, w_{k}(x)\right)\right) \\
& \cdot w_{k}(x) \geq 0,
\end{aligned}
$$

for all $x \in A_{k}^{-}$. Hence, on account of (193) and (195), from (191) we obtain the required estimate of (187).

(a) To prove this part, note that from Definition 40 it is clear that

$$
\left\|w_{k}\right\|_{W^{1, p}(\Omega)}^{p} \leq \mathscr{A}\left(W_{k}, W_{k}\right) .
$$

Let $f \in L^{p_{1}}(\Omega)$ and $g \in L^{q_{1}}(\partial \Omega)$ with

$$
\begin{aligned}
& p_{1}>\frac{p_{s}}{p_{s}-p}=\frac{N}{p}, \\
& q_{1}>\frac{q_{s}}{q_{s}-p}=\frac{N-1}{p-1},
\end{aligned}
$$

and let $B \subset \bar{\Omega}$ be any $\mu$-measurable set. We claim that there exists a constant $C \geq 0$ such that, for every $F \in X^{p_{1}, q_{1}}(\bar{\Omega}, \mu)$ and $\varphi \in W^{1, p}(\Omega)$, we have

$$
\left\|F \varphi 1_{B}\right\|_{1,1} \leq C\left\|\left|F\left\|\left.\right|_{p_{1}, q_{1}}\right\| \varphi\left\|_{W^{1, p}(\Omega)}\right\|\left\|\chi_{B}\right\|_{p_{3}, q_{3}},\right.\right.
$$

where $p_{3}$ and $q_{3}$ are such that $1 / p_{3}+1 / p_{1}+1 / p_{s}=1$ and $1 / q_{3}+1 / q_{1}+1 / q_{s}=1$. In fact, note that if $n \in \mathbb{N}$ and $p_{i}$, $q_{i} \in[1, \infty](i=1, \ldots, n)$ are such that

$$
\sum_{i=1}^{n} \frac{1}{p_{i}}=\sum_{i=1}^{n} \frac{1}{q_{i}}=1,
$$

and if $F_{i} \in X^{p_{i}, q_{i}}(\bar{\Omega}, \mu)(i=1, \ldots, n)$, then by Hölder's inequality

$$
\left.\left\|\prod_{i=1}^{n} F_{i}\right\|\right|_{1,1} \leq \prod_{i=1}^{n}\left\|\left|F_{i} \|\right|_{p_{i}, q_{i}} .\right.
$$

Since $W^{1, p}(\Omega) \hookrightarrow X^{p_{s}, q_{s}}(\bar{\Omega}, \mu)$, (198) follows immediately from (200) and claim (198) is proved. Next, it follows from (198) that

$$
\begin{aligned}
\int_{\bar{\Omega}} F W_{k} d \mu & =\left.\left\|F W_{k}\right\|\right|_{1,1}=\left.\left\|F W_{k} \chi_{A_{k}}\right\|\right|_{1,1} \\
& \leq\left.\left.\|F\|\right|_{p_{1}, q_{1}}\left\|\left|w_{k}\left\|\left.\right|_{W^{1, p}(\Omega)}\right\|\right| \chi_{A_{k}}\right\|\right|_{p_{3}, q_{3}},
\end{aligned}
$$

where we recall that $1 / p_{3}=\left(1-1 / p_{s}-1 / p_{1}\right)>(p-1) / p_{s}$ and $q_{3}<q_{s} /(p-1)$. Therefore, for every $k \geq 0$

$$
\begin{aligned}
& \mathscr{A}\left(U, W_{k}\right)-\mathscr{A}\left(V, W_{k}\right) \\
& \quad \leq\left.\|F\|\right|_{p_{1}, q_{1}}\left\|w_{k}\right\|_{W^{1, p}(\Omega)}\left\|\left|\chi_{A_{k}} \|\right|_{p_{3}, q_{3}},\right.
\end{aligned}
$$

which together with estimate (187) yields the desired inequality

$$
\begin{aligned}
C \mathscr{A}\left(W_{k}, W_{k}\right) & \leq \mathscr{A}\left(U, W_{k}\right)-\mathscr{B}\left(V, W_{k}\right) \\
& \leq\|\mid F\|_{p_{1}, q_{1}}\left\|w_{k}\right\|_{W^{1, p}(\Omega)}\left\|\chi_{A_{k}}\right\|_{p_{3}, q_{3}} .
\end{aligned}
$$

It follows from (196) and (203) that for every $k>0$

$$
\begin{aligned}
C\left\|w_{k}\right\|_{W^{1, p}(\Omega)}^{p} & \leq \mathscr{A}\left(W_{k}, W_{k}\right) \\
& \leq \mathscr{A}\left(U, W_{k}\right)-\mathscr{A}\left(V, W_{k}\right) \\
& \leq\left\|\left|F\left\|\left.\right|_{p_{1}, q_{1}}\right\| w_{k}\left\|_{W^{1, p}(\Omega)}\right\|\right| \chi_{A_{k}} \mid\right\|_{p_{3}, q_{3}} .
\end{aligned}
$$

Hence, for every $k>0,\left\|w_{k}\right\|_{W^{1, p}(\Omega)}^{p^{-1}} \leq C_{1}\left\|\left|\chi_{A_{k}} \|\right|_{p_{3}, q_{3}}\right.$. Using the fact $W^{1, p}(\Omega) \hookrightarrow X^{p_{s}, q_{s}}(\bar{\Omega}, \mu)$, we obtain, for every $k>0$, that

$$
\left.\left.\left\|\left|w_{k}\left\|\left.\right|_{p_{s}, q_{s}} ^{p-1} \leq C\right\|\right| F\right\|\right|_{p_{1}, q_{1}}\left\|\chi_{A_{k}}\right\|\right|_{p_{3}, q_{3}}
$$

Let $h>k$. Then $A_{h} \subset A_{k}$ and on $A_{h}$ the inequality $\left|w_{k}\right| \geq$ $(h-k)$ holds. Therefore

$$
\left.\left\|(h-k) \chi_{A_{h}}\right\|\right|_{p_{s}, q_{s}} ^{p-1} \leq C\left\|\left|F\left\|\left.\right|_{p_{1}, q_{1}}\right\|\left\|\chi_{A_{k}}\right\| \|_{p_{3}, q_{3}},\right.\right.
$$

which shows that

$$
\left.\left\|\left|\chi_{A_{h}}\left\|\left.\right|_{p_{s}, q_{s}} ^{p-1} \leq C\right\|\right| F\right\|\right|_{p_{1}, q_{1}}(h-k)^{-(p-1)}\left\|\chi_{A_{k}}\right\| \|_{p_{3}, q_{3}} .
$$

Let $C_{3}:=\left\||1 \bar{\Omega} \||_{p_{s}, q_{s}}\right.$ and

$$
\begin{aligned}
\delta & :=\min \left\{\frac{p_{s}}{p_{3}}, \frac{q_{s}}{p_{3}}\right\}>p-1, \\
\delta_{0} & :=\frac{\delta}{p-1}>1 .
\end{aligned}
$$


Then

$$
\begin{aligned}
\left\|C_{3}^{-p_{s} / p_{3}} \chi_{A_{k}}\right\|_{\Omega, p_{3}} & =\left\|C_{3}^{-1} \chi_{A_{k}}\right\|_{\Omega, p_{s}}^{p_{s} / p_{3}} \leq\left\|C_{3}^{-1} \chi_{A_{k}}\right\|_{\Omega, p_{s}}^{\delta} \\
& \leq\left.\left\|\chi_{A_{k}}\right\|\right|_{p_{s}, q_{s}} ^{\delta} C_{3}^{-\delta}, \\
\left\|C_{3}^{-q_{s} / q_{3}} \chi_{A_{k}}\right\|_{\partial \Omega, q_{3}} & =\left\|C_{3}^{-1} \chi_{A_{k}}\right\|_{\partial \Omega, q_{s}}^{q_{s} / q_{3}} \leq\left\|C_{3}^{-1} \chi_{A_{k}}\right\|_{\partial \Omega, q_{s}}^{\delta} \\
& \leq\left\|\chi_{A_{k}}\right\| \|_{p_{s}, q_{s}}^{\delta} C_{3}^{-\delta} .
\end{aligned}
$$

Choosing $C_{\Omega}:=C_{3}^{p_{s} / p_{3}-\delta}+C_{3}^{q_{s} / q_{3}-\delta}$, from (209) we have

$$
\left.\left\|\chi_{A_{k}}\right\|\right|_{p_{3}, q_{3}} \leq\left. C_{\Omega}\left\|\chi_{A_{k}}\right\|\right|_{p_{s}, q_{s}} ^{\delta}
$$

Therefore, combining (207) with (210), we get

$$
\begin{gathered}
\|\| \chi_{A_{h}}\left\|\left.\right|_{p_{s}, q_{s}} ^{p-1} \leq C\right\|\left|F\left\|\left.\right|_{p_{1}, q_{1}}(h-k)^{-(p-1)}\right\|\left\|\chi_{A_{k}}\right\| \|_{p_{s}, q_{s}}^{\delta}\right. \\
=C\left\||F \||_{p_{1}, q_{1}}(h-k)^{-(p-1)}\left[\left.\left\|\chi_{A_{k}}\right\|\right|_{p_{s}, q_{s}} ^{p-1}\right]^{\delta_{0}} .\right.
\end{gathered}
$$

Setting $\psi(h):=\left\|\left|\chi_{A_{h}} \|\right|_{p_{s}, q_{s}}^{p-1}\right.$ in Lemma 15, on account of (211), we can find a constant $C_{2}$ (independent of $F$ ) such that

$$
\left.\left\|\chi_{A_{K}}\right\|\right|_{p_{s}, q_{s}} ^{p^{-1}}=0 \quad \text { with } K:=\left.C_{2}\|F\|\right|_{p_{1}, q_{1}} ^{1 /(p-1)}
$$

This shows that $\mu\left(A_{K}\right)=0$, where $A_{K}=\{x \in \bar{\Omega}: \mid(u-$ $v)(x) \mid \geq K\}$. Hence, we have $|u-v| \leq K, \mu$, a.e. on $\bar{\Omega}$, so that

$$
\begin{aligned}
\|\mid U-V\|_{\infty}^{p-1} & \leq\left. C_{2}\|F\|\right|_{p_{1}, q_{1}} \\
& =C_{2}\left(\|f\|_{p_{1}, \Omega}+\|g\|_{q_{1}, \partial \Omega}\right),
\end{aligned}
$$

which completes the proof of part (a).

(b) To prove this part, instead of (196) and (198), one uses $\left\|W_{k}\right\|_{\mathscr{V}_{1}}^{p} \leq \mathscr{A}\left(W_{k}, W_{k}\right)$ and $\left\|\left|F \varphi 1_{B} \|\right|_{1,1} \leq\right.$ $\left.C\left\|\left|F\left\|\left.\right|_{p_{1}, q_{1}}\right\| \varphi\left\|_{W^{1, p}(\Omega)}\right\|\right| \chi_{B}\right\|\right|_{p_{3}, q_{3}}$, where $p_{3}$ and $q_{3}$ are such that $1 / p_{3}+1 / p_{1}+1 / p_{s}=1$ and $1 / q_{3}+1 / q_{1}+1 / p_{t}=1$, and the embedding $\mathscr{V} \hookrightarrow \mathscr{V}_{1} \hookrightarrow X^{p_{s}}, p_{t}(\bar{\Omega}, \mu)$. The remainder of the proof follows as in the proof of part (a).

We conclude this section with the following example.

Example 48. Let $p \in[2, \infty), b: \partial \Omega \rightarrow(0, \infty)$ be a strictly positive and $\sigma$-measurable function and let

$$
\beta(x, \xi):=b(x)|\xi|^{p-2} \xi, \quad \xi \in \mathbb{R} .
$$

Then, it is easy to verify that $\beta$ satisfies Assumptions 38, 39, and 44 (see, e.g., [25, Example 4.17]).

\section{Conflicts of Interest}

The authors declare that they have no conflicts of interest.

\section{References}

[1] C. G. Gal, M. Grasselli, and A. Miranville, "Nonisothermal Allen-Cahn equations with coupled dynamic boundary conditions," in Nonlinear phenomena with energy dissipation, vol. 29 of GAKUTO Internat. Ser. Math. Sci. Appl., pp. 117-139, Gakko Tosho, Tokyo, Japan, 2008.

[2] C. G. Gal and A. Miranville, "Uniform global attractors for nonisothermal viscous and non-viscous Cahn-Hilliard equations with dynamic boundary conditions," Nonlinear Analysis. Real World Applications. An International Multidisciplinary Journal, vol. 10, no. 3, pp. 1738-1766, 2009.

[3] S. Agmon, A. Douglis, and L. Nirenberg, "Estimates near the boundary for solutions of elliptic partial differential equations satisfying general boundary conditions I," Communications on Pure and Applied Mathematics, vol. 12, pp. 623-727, 1959.

[4] C. G. Gal, G. R. Goldstein, J. A. Goldstein, S. Romanelli, and M. Warma, "Fredholm alternative, semilinear eliptic problems, and Wentzell boundary conditions," http://arxiv.org/abs/1311.3134.

[5] L. Hörmander, Linear partial differential operators, SpringerVerlag, Berlin, Germany, 1976.

[6] J. Peetre, "Another approach to elliptic boundary problems," Communications on Pure and Applied Mathematics, vol. 14, pp. 711-731, 1961.

[7] M. I. Vishik, "On general boundary problems for elliptic differential equations," Trudy Moskovskogo Matematicheskogo Obshchestva, vol. 1, pp. 187-246, 1952.

[8] E. M. Landesman and A. C. Lazer, "Nonlinear perturbations of linear elliptic boundary value problems at resonance," Journal of Applied Mathematics and Mechanics, vol. 19, pp. 609-623, 1970.

[9] H. m. Brézis and A. Haraux, "Image d'une somme d’opérateurs monotones et applications," Israel Journal of Mathematics, vol. 23, no. 2, pp. 165-186, 1976.

[10] H. m. Brézis and L. Nirenberg, "Image d'une somme d'opérateurs non linéaires et applications," Comptes Rendus Mathématique. Académie des Sciences. Paris, vol. 284, no. 21, pp. A1365-A1368, 1977.

[11] P. h. Bénilan and M. G. Crandall, "Completely accretive operators," in Lecture Notes in Pure and Applied Mathematics, Semigroup theory and evolution equations (Delft, 1989), Dekker, New York, NY, USA, 1991.

[12] H. Brézis, Opérateurs maximaux monotones et semi-groupes de contractions dans les espaces de hilbert, American Elsevier Publishing Co., Inc., New York, NY, USA, 1973.

[13] G. J. Minty, "Monotone (nonlinear) operators in Hilbert space," Duke Mathematical Journal, vol. 29, pp. 341-346, 1962.

[14] G. J. Minty, "On the solvability of nonlinear functional equations of 'monotonic' type," Pacific Journal of Mathematics, vol. 14, pp. 249-255, 1964.

[15] R. E. Showalter, Monotone operators in Banach space and nonlinear partial differential equations, vol. 49 of Mathematical Surveys and Monographs, American Mathematical Society, Providence, RI, USA, 1997.

[16] J. Necas, Les Méthodes Directes en Théorie des Équations Elliptiques, Masson, Paris, France, 1967.

[17] V. G. Maz'ya and S. V. Poborchi, Differentiable functions on bad domains, World Scientific Publishing Co., Inc., 1997.

[18] R. A. Adams, Sobolev Spaces. Pure and Applied Mathematics, Academic Press, New York, NY, USA, 1975.

[19] M. M. Rao and Z. D. Ren, Theory of Orlicz Spaces, Monographs and Textbooks in Pure and Applied Mathematics, Marcel Dekker, New York, NY, USA, 1991. 
[20] M. M. Rao and Z. D. Ren, Applications Of Orlicz Spaces, vol. 250 of Monographs and Textbooks in Pure and Applied Mathematics, CRC Press, Marcel Dekker, Inc., 2002.

[21] E. DiBenedetto, Degenerate Parabolic Equations, Springer, New York, NY, USA, 1993.

[22] M. K. Murthy and G. Stampacchia, "Boundary value problems for some degenerate-elliptic operators," Annali di Matematica Pura ed Applicata. Serie Quarta, vol. 80, pp. 1-122, 1968.

[23] V. G. Maz'ya, Sobolev spaces, Springer Series in Soviet Mathematics, Springer-Verlag, Berlin, Germany, 1985.

[24] H. Attouch, G. Buttazzo, and G. Michaille, Variational Analysis in Sobolev and BV Spaces. Applications to PDEs and optimization, vol. 6 of MPS/SIAM Series on Optimization, Society for Industrial and Applied Mathematics (SIAM), Philadelphia, PA, USA, 2006.

[25] M. Biegert and M. Warma, "The heat equation with nonlinear generalized Robin boundary conditions," Journal of Differential Equations, vol. 247, no. 7, pp. 1949-1979, 2009.

[26] M. Warma, "The ROBin and Wentzell-ROBin Laplacians on LIPschitz domains," Semigroup Forum, vol. 73, no. 1, pp. 10-30, 2006.

[27] M. Biegert and M. Warma, "Some quasi-linear elliptic equations with inhomogeneous generalized Robin boundary conditions on "bad" domains", Advances in Differential Equations, vol. 15, no. 9-10, pp. 893-924, 2010.

[28] P. Drábek and J. Milota, Methods of Nonlinear Analysis. Applications to Differential Equations, Birkhäuser Advanced Texts, Birkhäuser, Basel, Switzerland, 2007. 


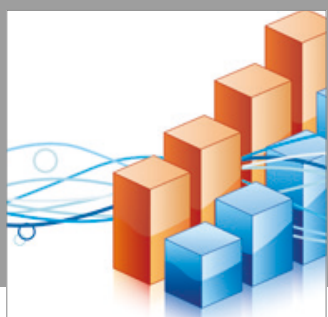

Advances in

Operations Research

vatersals

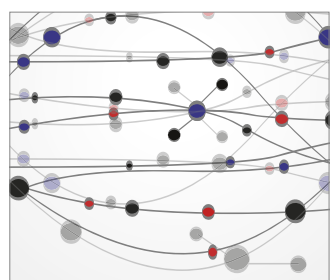

\section{The Scientific} World Journal
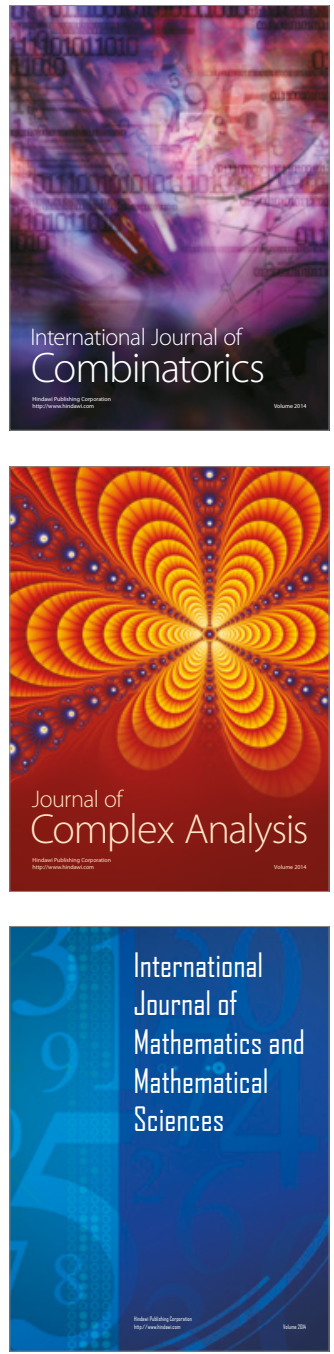
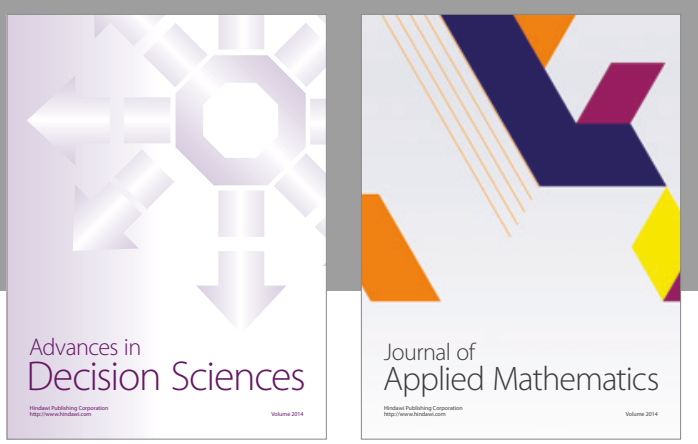

Algebra

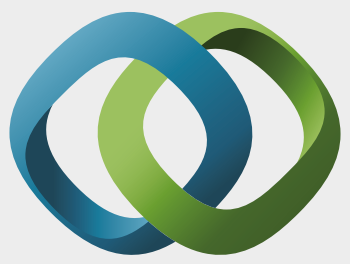

\section{Hindawi}

Submit your manuscripts at

https://www.hindawi.com
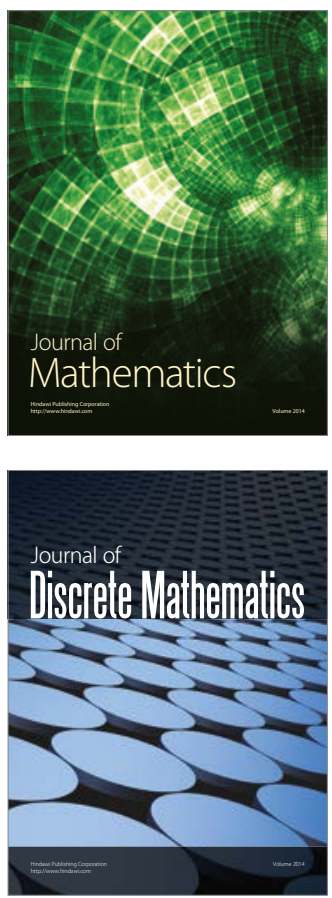

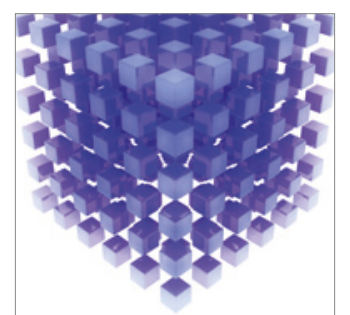

Mathematical Problems in Engineering
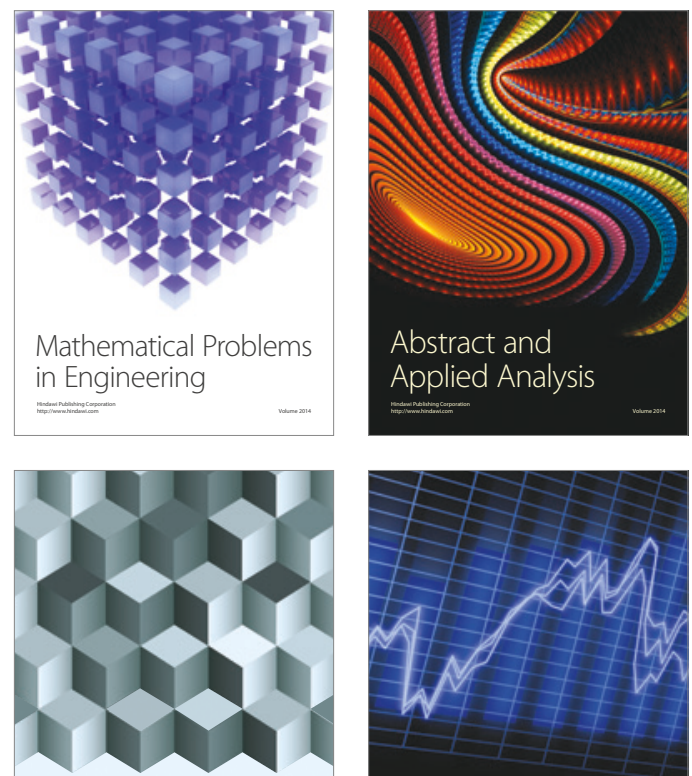

Journal of

Function Spaces

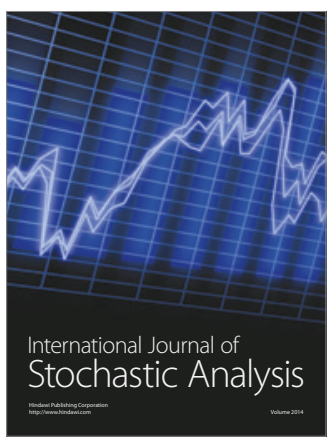

Probability and Statistics
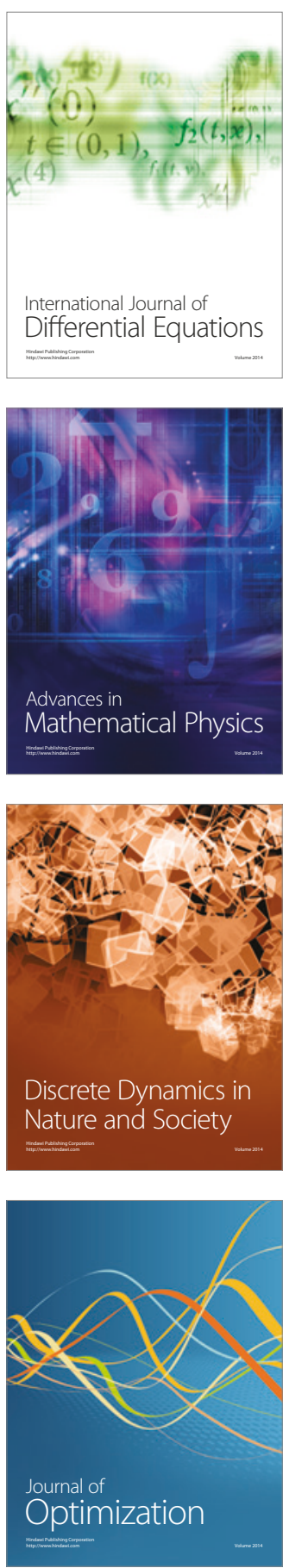